\title{
THESIS
}

\section{DEMONSTRATION OF A COMPACT 100 Hz, 0.1 J, DIODE-PUMPED PICOSECOND LASER}

\author{
Submitted by \\ Alden Curtis \\ Department of Electrical and Computer Engineering
}

\author{
In partial fulfillment of the requirements \\ For the Degree of Master of Science \\ Colorado State University \\ Fort Collins, Colorado
}

Fall 2011

Masters Committee:

Advisor: Jorge Rocca

Azer Yalin

Diego Krapf 


\section{ABSTRACT \\ SOLID STATE DIODE-PUMPED SHORT PULSE LASER}

In this work I present an all laser diode pumped chirped pulse amplification laser system that is capable of producing $100 \mathrm{~mJ}$ laser pulses at $100 \mathrm{~Hz}$ repetition rate with durations of under 5 ps. The primary focus of this work consists of the development of two amplification stages that boost the temporally stretched pulses from a few hundred picoJoules to more than $100 \mathrm{~mJ}$. The first amplifier is a Yb:YAG based regenerative amplifier operated at room temperature, which amplifies the pulses by a factor of about $10^{6}$. The second stage is a multi-pass, Yb:YAG based amplifier, which is operated at cryogenic temperatures, and further amplifies the pulses by a factor of about 100 . This is the first time a combination of room temperature and cryogenically cooled Yb:YAG amplifiers has been demonstrated. The room temperature pre-amplifier maintains more bandwidth than in the cryogenic case for increased compressibility. The cryogenic cooling of the power amplifier allows for increased heat dissipation and decreased saturation intensity for efficient operation. The optical efficiency of this amplifier is higher than that of other diode-pumped systems of comparable energy. 


\section{Contents}

1 Introduction 1

1.1 Solid State Lasers . . . . . . . . . . . . . . . . . . . . . . . 1

1.2 Pulsed Solid State Lasers . . . . . . . . . . . . . . . . . . . . 1

1.2.1 Short Pulse Oscillators . . . . . . . . . . . . . . . 2

1.2.2 Chirped Pulse Amplification . . . . . . . . . . . . 5

1.2 .3 Diode Pumping . . . . . . . . . . . . . . . . . 8

1.3 Active Media . . . . . . . . . . . . . . . . . . . . . 9

1.3.1 $Y b^{3+}$ Atomic Structure . . . . . . . . . . . . . . 10

1.3.2 Host Material . . . . . . . . . . . . . . . . . . . . . . . 12

1.3.3 Temperature Dependence of Yb:YAG Characteristics . . . . 14

1.4 Cooling Geometry . . . . . . . . . . . . . . . . . . . . . . . . . 19

1.5 Diode-Pumped Yb:YAG CPA Systems _ . . . . . . . . . . 20

1.5.1 State of the Art Room Temperature CPA's . . . . . . . . . . 21

1.5.2 State of the Art Cryogenic Temperature CPA's . . . . . . . 22

1.6 conclusion . . . . . . . . . . . . . . . . . . . . . . . 24

2 System Overview 26

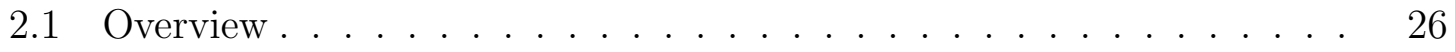

2.2 Semi-Conductor Laser Diode Pumps _ . . . . . . . . . . . . . 28

2.3 Gain Media . . . . . . . . . . . . . . . . . . . . . . . . . . . 31 
2.4 Isolation Between Stages . . . . . . . . . . . . . . . . . . . . 33

3 Oscillator and Stretcher 35

3.1 Oscillator . . . . . . . . . . . . . . . . . . . . . 35

3.2 Stretcher . . . . . . . . . . . . . . . . . . 40

4 Regenerative Amplifier $\quad 44$

4.1 Description of a Regenerative Amplifier . . . . . . . . . . . . . . . . . 44

4.2 Description of Cavity and Optics . . . . . . . . . . . . 45

4.3 Regenerative Amplifier Results . . . . . . . . . . . . . . . . . 48

4.3.1 Future Improvements to 1st Stage Amplifier . . . . . . . . . . 54

4.3.2 Final Comments . . . . . . . . . . . . . . 57

$5 \quad$ Multi-pass amplifier $\quad 58$

5.1 Amplifier Description and Optics . . . . . . . . . . . 58

5.2 Multi-pass Amplifier Performance . . . . . . . . . . . . . 61

5.3 Compressor .......................... 62

6 Conclusion $\quad 65$ 


\section{Chapter 1}

\section{Introduction}

\subsection{Solid State Lasers}

Since the postulation and subsequent demonstration of the laser in 1960, a large number of different media for generating lasers have been demonstrated. These include solid state lasers, gas discharge (plasma) lasers, semiconductor diode lasers, dye lasers, and others. Of particular interest to this work are solid state lasers, where the gain material is a crystal or glass, or any solid material that is transparent to the wavelength of interest. This material is doped to some degree with the actual lasing element. One of the main features of this type of laser is that the population inversion in the active media is created by optical pumping with flashlamps or other lasers. A diverse number of solid state lasers have been demonstrated over time from high power continuous wave lasers to ultrafast pulse lasers. In fact, a solid state laser, the ruby laser, was the first laser to be demonstrated [1, 2].

\subsection{Pulsed Solid State Lasers}

Interest in the development of short pulse, high peak power lasers is important to a number of industrial and scientific applications including biomedical processes[3], 
material science [4], inertial confinement fusion [5], and table-top soft x-ray laser generation [6]. In order for short pulse lasers to be useful for many applications, amplification of the pulse energy is necessary. Mode locked oscillators create ultrashort pulses with low per pulse energy. These pulses are then sent to amplifiers to increase the energy. These amplifiers are implemented by optically pumping a material until a population inversion is created. The laser pulses pass through the material, extract the energy, and are amplified. Many considerations affect how the amplifier system will be implemented such as gain narrowing, wavefront degradation, dispersion effects, and non-linear affects [7].

The two most common methods for generating high peak power laser pulses are qswitching and mode-locking a laser cavity. Although q-switching can generate higher energy pulses without amplification, only a mode-locked laser can create pulses of less than a few hundred picoseconds, and down into the femtosecond regime [7]. The short time scale and the resultant peak power, which can easily reach TerraWatts, make mode-locked lasers useful for entirely different applications than continuous wave (cw) or quasi-cw lasers, such as probing events on an atomic time scale or generating hot dense plasmas [8].

\subsubsection{Short Pulse Oscillators}

The term mode-locking refers to locking the phase between the longitudinal cavity modes, which simply means keeping a constant and consistent phase between all the modes. If such a relationship between the modes can be maintained, interference between all of the separate standing waves will result in an extremely short temporal duration, or ultrafast, optical pulse. For a given optical resonator length, a broader emission bandwidth of the gain material will result in a larger number of longitudinal modes. The interference of a large number of modes allows for the generation of short pulses, so when seeking to create ultrafast pulses a broadband gain media is essential. 
In a passively mode-locked oscillator a saturable absorber is used to initiate and maintain mode-locked behavior. A saturable absorber is a material that will absorb energy until the absorption threshold is reached and then abruptly cease. Some low level of oscillation depending on the absorption saturation is kept from meeting the requirements of at least unity round trip gain. If, however, some spike in the noise rises above this threshold, this spike starts to be preferentially amplified and eventually becomes a short pulse travelling around the cavity. The absorber must also have fast relaxation time to damp out non-primary pulse oscillations which deplete the gain. Saturable absorber bandwidth is also important in determining the shortest possible pulse duration [9].

Short pulses experience temporal dispersion due to the different spectral components experiencing a varying index of refraction in a material. Material dispersion for many typical solid state laser wavelengths is usually positive dispersion, where the longer wavelengths travel slightly faster. This effect is problematic in the gain material of a mode-locked oscillator as it increases the pulse duration and interrupts the mode-locking process. Positive dispersion must be compensated for by negative dispersion in order to maintain short pulse durations. Dispersion effects can be calculated using a Taylor expansion of the frequency dependent spectral phase $\varphi(\omega)$ of the pulse, which takes the form

$$
\varphi(\omega)=\varphi_{0}+\varphi_{1}\left(\omega-\omega_{0}\right)+\frac{1}{2} \varphi_{2}\left(\omega-\omega_{0}\right)^{2}+\frac{1}{6} \varphi_{3}\left(\omega-\omega_{0}\right)^{3}+\ldots=\sum_{n=1}^{\infty} \frac{\varphi_{n}}{n !} \cdot\left(\omega-\omega_{0}\right)^{n}
$$

where

$$
\left.\varphi_{n} \equiv \frac{d^{n} \varphi(\omega)}{d \omega^{n}}\right|_{\omega=\omega_{0}}
$$

Here $\varphi_{0}$ through $\varphi_{n}$ represent the different possible orders of dispersion, $\omega_{0}$ is the center frequency and $\omega$ is the frequency of interest. It can be seen from the 
equation that there are many possible orders of dispersion. $\varphi_{0}$ and $\varphi_{1}$ represent the initial phase and group delay and therefore have no effect on the temporal pulse shape. $\varphi_{2}$ linearly stretches the pulse duration, and the higher orders distort the pulse in more complicated ways. Typically all of the orders cannot be compensated for, so measures are taken to reduce the orders that have the strongest pulse temporal distortion effects, orders 2 and 3.

This is most typically accomplished using a prism pair in the oscillator which compensates the dispersion by causing the different frequency components to take slightly different path lengths through a certain section of the cavity. By choosing the correct distance for these prisms the correct amount of negative dispersion can be imparted to the pulse resulting in nearly transform limited pulses at the output of the cavity. This spacing can be found for some desired second order dispersion to be compensated for, $\varphi_{2}(\omega)$ and third order, $\varphi_{3}(\omega)$ from the equations

$$
\begin{aligned}
& \varphi_{2}(\omega)=D_{2 L} L+D_{2 x} x \\
& \varphi_{3}(\omega)=D_{3 L} L+D_{3 x} x
\end{aligned}
$$

where $\mathrm{L}$ is the prism separation, $\mathrm{x}$ is the prism insertion, and the $\mathrm{D}$ coefficients are

$$
\begin{gathered}
D_{2 L}=-\frac{2 \lambda}{\pi c^{2}} \lambda\left(\frac{d n}{d \lambda}\right)^{2} \\
D_{2 x}=-\frac{2 \lambda}{\pi c^{2}} \lambda^{2} \frac{d^{2} n}{d \lambda^{2}} \frac{1}{n} \\
D_{3 L}=-\frac{3 \lambda}{\pi^{2} c^{3}}\left(\lambda \frac{d n}{d \lambda}\right)\left(\lambda \frac{d n}{d \lambda}+\lambda^{2} \frac{d^{2} n}{d \lambda^{2}}\right) \\
D_{3 x}=-\frac{3 \lambda^{2}}{2 \pi^{2} c^{3}}\left(\lambda^{2} \frac{d^{2} n}{d \lambda^{2}}+\frac{\lambda^{3}}{3} \frac{d^{3} n}{d \lambda^{3}}\right) \frac{1}{n}
\end{gathered}
$$

where $\lambda$ is wavelength, $\mathrm{c}$ is the speed of light, and $\mathrm{n}$ is the refractive index. These equations are simply derived from geometrical ray tracing of the different frequency 


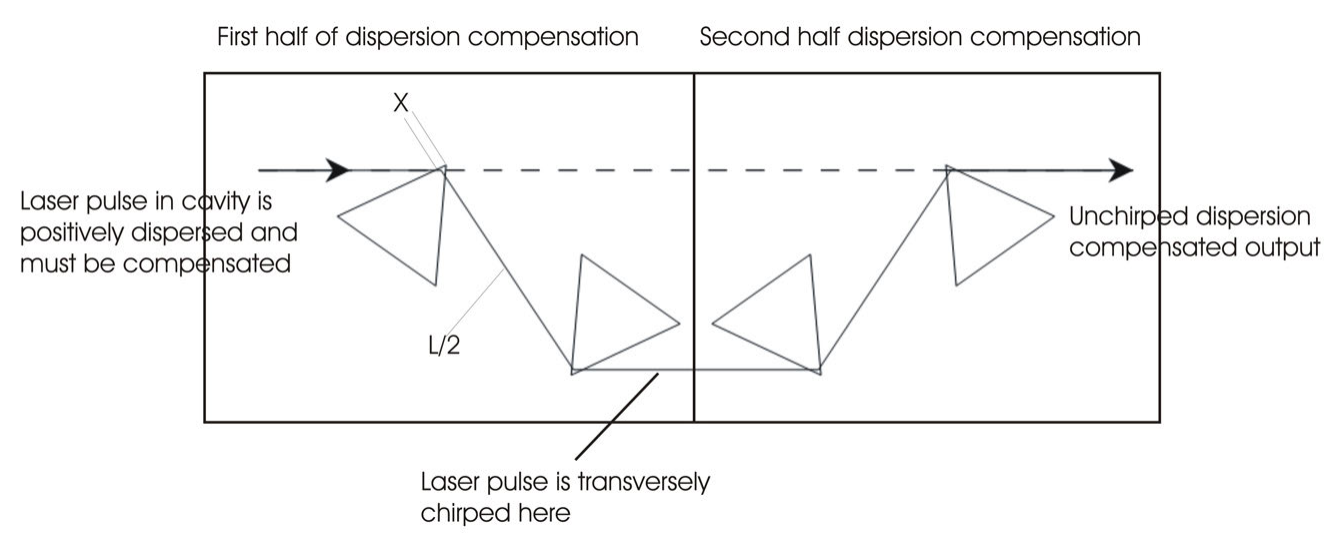

Figure 1.1: Typical prism dispersion compensation within an oscillator cavity using four prisms to avoid spatial chirp at the output.

elements through two prisms. These equations indicate that such dispersion compensation can be practically implemented in a cavity only a few meters in length.

Typically, in order to avoid spatial chirp which is effectively spreading the frequency components across the transverse profile of the beam, an arrangement such as the one shown in Fig. 1.1 is used with 4 prisms instead of two [9]. In this arrangement the second prism pair reverses the spatial chirp that has been added by the first prism pair, and the separation of each pair is halved. Another possible option is to design a mirror that will add negative dispersion to the pulse. Because such a mirror would only longitudinally disperse the pulse, no transverse spatial chirp would be added to the beam. This technique is further investigated below.

\subsubsection{Chirped Pulse Amplification}

Mode-locked oscillators typically produce pulses with nanoJoules of energy. To increase the pulse energy, a scheme must be implemented where the non-linear effects and optical damage associated with such high peak intensities do not interrupt the amplification process. The most common way that very intense laser pulses are generated currently is chirped pulse amplification (CPA) of mode-locked, solid state laser systems. These systems work by purposefully temporally dispersing the fre- 


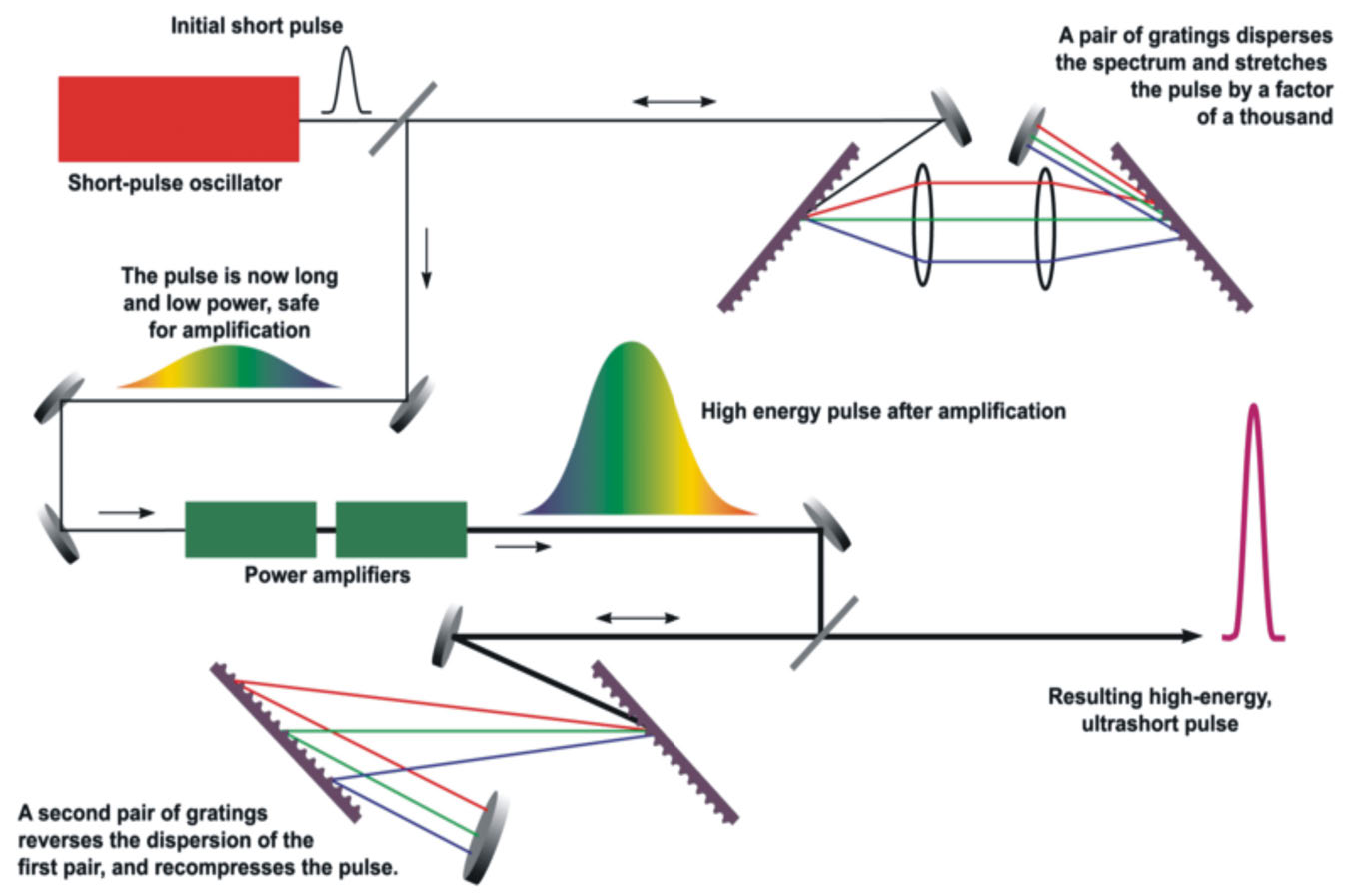

Figure 1.2: Typical layout for a chirped pulse amplification system, although there are many additional structures that can vary from system to system, shown here are the essential pieces. [10]

quency components of the fast laser pulse, so called adding chirp to the pulse, which increases the temporal pulse duration and results in a decreases of the peak pulse intensity. These stretched pulses can be amplified in intensity over many orders of magnitude without having to worry about unwanted non-linear effects. After amplification to the desired pulse energy, the laser pulses are recompressed by temporally overlapping the frequency components once again, thereby obtaining much higher peak intensities ([8]). A CPA system is schematically shown in fig 1.2 .

In this scheme, ultrashort pulses are created in a mode-locked laser oscillator. The ultrashort pulses exiting the oscillator are stretched in time using diffraction gratings and an imaging system. This is the same concept that is used for dispersion compensation in the oscillator except diffraction gratings have much greater angular dispersion than prisms and so are able to add a lot more dispersion to the pulse [9]. Additionally the dispersion added to the pulse can be made positive using an imaging 
system between the gratings.

There are several ways that the stretcher can be implemented. The most common arrangements are Martinez and Offner stretchers. Martinez was the first to suggest the use of grating based stretchers, although currently stretchers in the original Martinez configuration are the least commonly used, because Martinez stretchers use lenses rather than curved mirrors as imaging components. Lenses result in more aberrations to the pulse front and can seriously distort ultrafast pulses [11]. Similarly to the prism pair, the amount of positive or negative group delay dispersion added to the pulse can be controlled based on certain geometric properties of the stretcher.

A Martinez stretcher is shown in Fig. 1.3. Much like a pulse compressor or prism pair, different spectral components will take a different path length through the stretcher. In order to impart positive dispersion however, the distance between the gratings has to be negative, which can be seen from the expression for 2nd order dispersion $\varphi_{2}(\lambda)$ as a function of grating separation, L, for any grating compressor/stretcher pair

$$
\varphi_{2}(\lambda)=\frac{L(\lambda / d)}{c d\left[1-(\lambda / d-\sin \gamma)^{2}\right]}
$$

where $\lambda$ is wavelength, $\mathrm{c}$ is the speed of light, $\mathrm{d}$ is grating line density, and gamma is the incident angle of the pulse impinging on the grating [12]. The imaging system is used to image the first grating behind the second grating making the distance effectively negative. This condition is satisfied so long as the distances of the gratings to the telescope, $\mathrm{X}$ in Fig. 1.3, are each less than the focal length for the lenses.

As seen in fig 1.2, the pulses are sent through a number of amplification stages to increase the energy after stretching. The geometries for solid state laser amplifiers vary greatly depending on material and energy expectations. There are different amplifier geometries that vary from single-pass to multi-pass to cavity based. The 


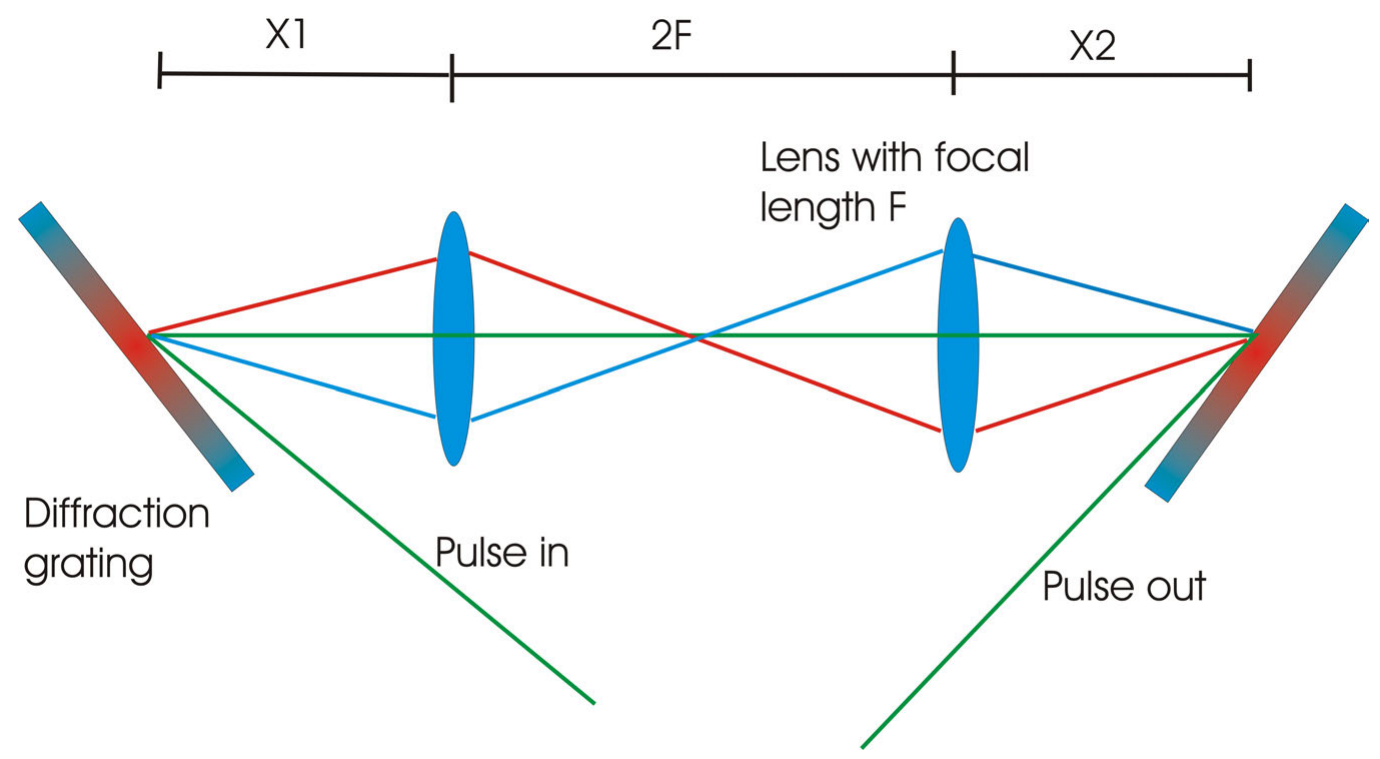

Figure 1.3: Schematic for Martinez grating stretcher.

choice of amplifier configuration depends greatly on shape of the gain media itself, where long rods and slabs are more likely to be single pass, and thinner transmission or reflection based gain media are more likely to be multipass. Often a combination of different amplifier arrangements is used [8].

After amplification the pulses are recompressed typically using a grating compressor. Here again the concept of different spectral component path length is used, but no lenses are necessary. The frequency components are angularly dispersed and sent through different physical path lengths in order to re-overlap them longitudinally. This produces a high energy ultrashort pulse.[9]. To avoid large energy loss in the compressor high efficiency gratings must be selected. In contrast, low efficiency gratings in the stretcher are of little importance because the lost energy can quickly be regained in the amplifiers.

\subsubsection{Diode Pumping}

Classically, most CPA laser systems use flashlamp pumping of the gain material. Particularly Ti:Sapphire CPA systems must use flashlamp pumped, Neodymium based 
lasers which are frequency doubled as a pump source, because of the short upper laser level lifetime of the material. However flash lamp pumping is inefficient for a few reasons. First flashlamps emit a very broad spectrum and waste most of the light they produce. Not only is this light not absorbed, a fraction of it turns into heat limiting the repetition rate at which that the lamp can be operated [13]. The wall plug efficiency of flashlamps is lower than laser diodes, the other widely accepted pump source for solid state lasers, reaching at most 40 percent for Krypton lamps [14].

It is the increased pumping efficiency allowed by semiconductor laser diodes that make them a more appropriate pump source for increasing the repetition rate of solid state CPA systems. Laser diodes are highly optically efficient, capable of pumping a single transition in a lasing material. They are also more electrically efficient than flashlamps, usually converting over 50 percent of the input electrical power to optical power. They are a much more directional source than flashlamps making pump geometries simpler, and are able to deliver higher average powers in a compact unit.

The major drawback of using laser diode pumps is that they deliver a lower peak power than is available from flashlamp pumping. Thus they are mostly used for pumping cw lasers or gain media with long upper level lifetimes, such as $Y b^{3+}$ based gain media which have millisecond lifetimes [13]. Commercially available high power diodes are available at 980 and $940 \mathrm{~nm}$ wavelengths that correspond to $Y b^{3+}$ absorption resonances. This makes diode lasers an ideal pump sources for $Y b^{3+}$ based CPA's. The features of the $Y b^{3+}$ ion and Yb:YAG are discussed in the next sections.

\subsection{Active Media}

There are many features of the different lasant atoms and ions to take into account when designing a solid state laser cavity or amplifier. Firstly, the wavelength and 
bandwidth will most likely vary widely depending on the application. How efficiently the host and lasant can be operated are important for high power applications. The efficiency of the active material will depend on atomic properties and energy structure. Host materials will have different efficiencies depending mostly on their thermal characteristics. The host response to different levels of doping can affect material stress or concentration quenching. Higher doping concentration allows for more energy storage and can affect high power and energy operation. A long upper laser level lifetime will also allow more energy storage. Finally the method for creating a population inversion has to be considered, depending on material and application.

Many of the most common solid state lasant ions emit in the near infrared. Some of these include Ytterbium, Neodymium, Erbium, and Titanium which lase at wavelengths centered around $1030 \mathrm{~nm}, 1060 \mathrm{~nm}, 1550 \mathrm{~nm}$, and $800 \mathrm{~nm}$ respectively. These different materials are used for a variety of applications. Ytterbium and Neodymium, which typically use glass or Yttrium Aluminum Garnet (YAG) as the host material, are used mostly for high power applications, whether pulsed or continuous wave operation is desired. Erbium has been utilized in optical communications applications, particularly as the dopant in fiber amplifiers. The emission spectrum of Titanium doped Sapphire can be tuned over a range of several hundred nanometers, centered around $800 \mathrm{~nm}$, and its broad bandwidth also allows for generation of extremely short, high peak power pulses in mode-locked lasers [13].

\subsection{1 $Y b^{3+}$ Atomic Structure}

The $Y b^{3+}$ ion has recently become a more popular choice for the laser material in systems that primarily use Neodymium. Both Ytterbium and Neodymium ions lase at 1 micron spectrally, and can be used as the gain media in mode-locked lasers. However, The $Y b^{3+}$ ion has a long upper level lifetime of almost 1 ms which makes laser diode pumping an important alternative to flashlamp pumping. Flashlamp 
Ytterbium

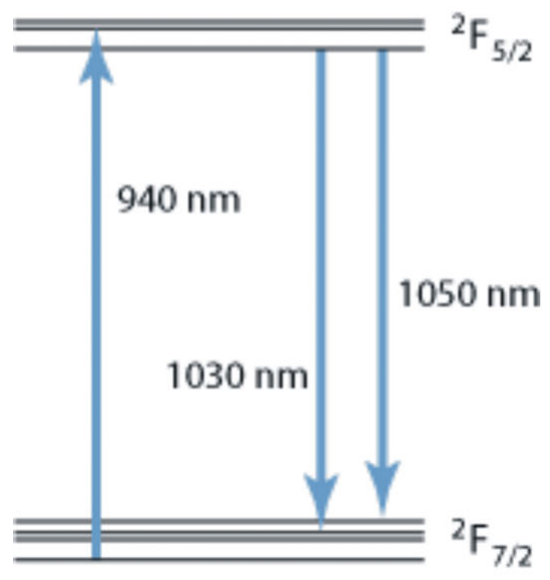

Neodymium

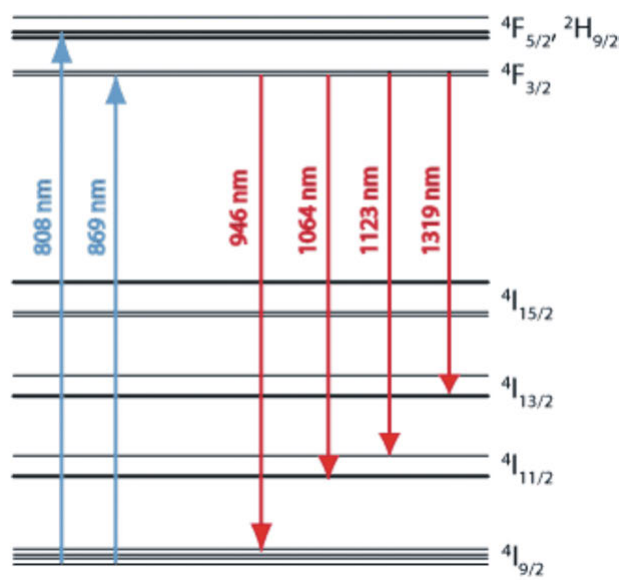

Figure 1.4: Energy level diagram for Ytterbium and Neodymium. The relatively simple electronic structure of Yb:YAG eliminates the possibility of a number of parasitic atomic processes that would reduce the gain of the laser line. The electronic structure of Neodymium is included for comparison. [16]

pumping is usually necessary for Neodymium which has an upper level lifetime of 230 $\mu s$ [7]. Additionally, Ytterbium has a significantly simpler electronic structure. An energy level diagram of Nd:YAG has been included for comparison of the simplicity of the energy level structure of $Y b^{3+}$ in Fig. 1.4.

The electronic structure of the $Y b^{3+}$ ion is comprised of basically two levels with all of the lasing dynamics occurring between various manifolds of the ${ }^{2} F_{7 / 2}$ and ${ }^{2} F_{5 / 2}$ levels as shown in Fig. 1.4. This eliminates parasitic processes which have been observed with Neodymium, which reduce gain in the material including excited state absorption, upconversion, and cross relaxation [15]. These quantum processes basically represent different possible de-excitation paths that ions excited to the upper laser level can take to leave that energy state. The ions available for stimulated emission are reduced by these processes, and so is the gain.

One can also observe from looking at the absorption and emission spectra of Ytterbium doped YAG (Yb:YAG) in fig. 1.5, that the absorption and emission wavelengths are very close together spectrally. This results in a very low quantum defect, q, 


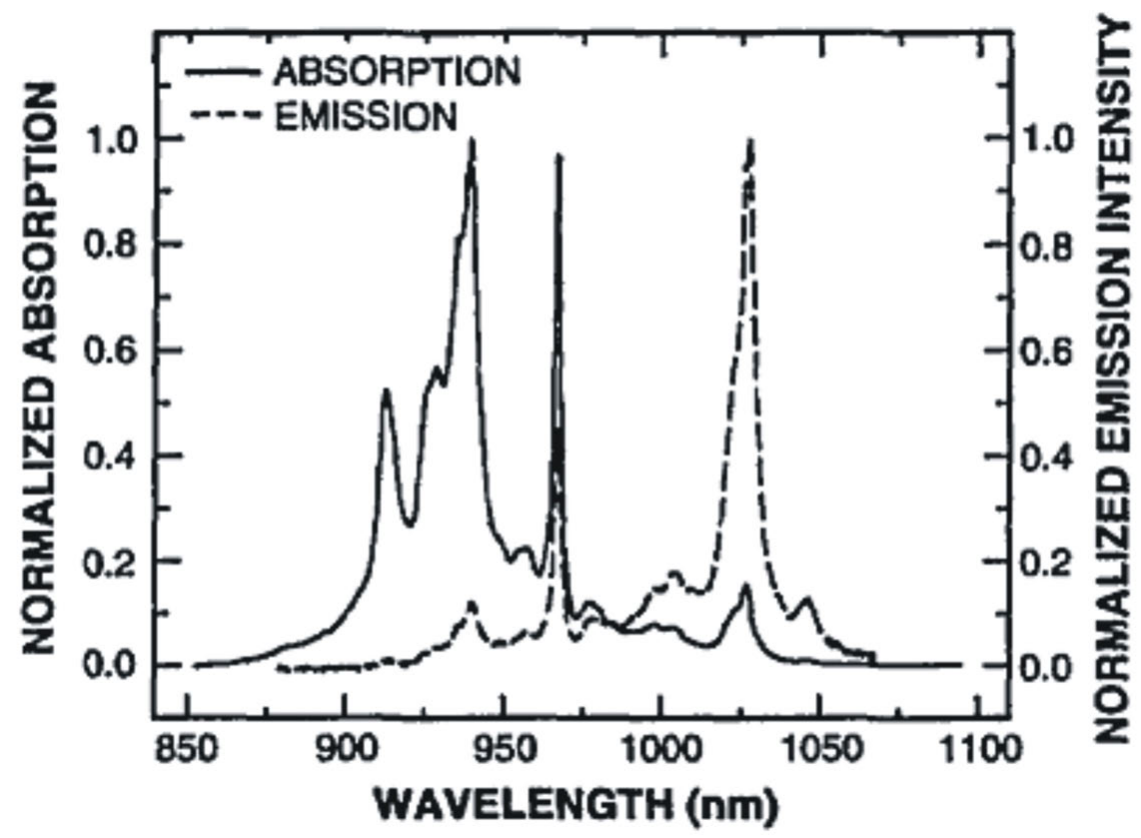

Figure 1.5: Yb:YAG emission and absorption spectrum [17]

$$
q=h \nu_{\text {pump }}-h \nu_{\text {laser }}
$$

in the material where $\nu_{\text {pump }}$ is the pump frequency, $\nu_{\text {laser }}$ is the emission frequency of the laser, and $\mathrm{h}$ is Plancks constant. In the case of Yb:YAG the optical efficiency that would result from only the quantum defect is close to 92 percent. This high optical efficiency is one of the primary reasons this particular material is well suited to high power operation. The excess pump energy determined by the quantum defect relaxes through phonon decay which causes heating of the gain material which is a challenge for engineering high average power laser systems [13].

\subsubsection{Host Material}

There are a number of glass and crystal structures that can be used as a host for the $Y b^{3+}$ ion which exhibit different properties. Due to the inhomogeneous molecular 
structure of glass, glass hosts are typically significantly more spectrally broadband than the crystalline hosts. this directly affects the stimulated emission cross section, $\sigma_{\text {stim }}$

$$
\sigma_{\text {stim }}=\frac{A_{21} \lambda^{2}}{(8 \pi) g(\nu)}
$$

which determines the small signal gain for the laser, where $A_{21}$ is the laser upper level lifetime, $\lambda$ is the wavelength of the laser transition, and $g(\nu)$ is the linewidth function. Because $g(\nu)$ is inversely related to the bandwidth of the transition it can be observed that a glass host will also display a decreased stimulated emission cross section and thus reduced small signal gain, compared with crystal hosts. Some of the various $\mathrm{Yb}^{3+}$ ion crystal hosts that have been observed lasing are $Y_{3} A_{5} \mathrm{O}_{12}$, $\mathrm{Lu}_{3} \mathrm{Al}_{5} \mathrm{O}_{12}, \mathrm{YAlO}_{3}, \mathrm{LiYF}_{4}, \mathrm{LiLuF}_{4}, \mathrm{BaY}_{2} \mathrm{~F}_{8}, \mathrm{KGd}(\mathrm{WO})_{2}$, and $\left.\mathrm{KY}(\mathrm{WO})_{4}\right)_{2}$. These different hosts exhibit a wide variety of qualities including different gain, thermal, and spectral characteristics [18].

It is instructive to compare some of the different host laser qualities such as bandwidth, quantum defect, and upper laser level lifetime. Some of these features can be seen in table 1.6 for different $\mathrm{Yb}$ hosts. In the case of Ytterbium doped $Y_{3} A l_{5} O_{12}$ (Yb:YAG), the quantum defect is around 8 percent, and the upper level lifetime is around $1 \mathrm{~ms}$. Ytterbium doped $\mathrm{LiYF}_{4}(\mathrm{Yb}: \mathrm{YLF})$ has a quantum defect of closer to 10 percent and an upper level lifetime of closer to $2 \mathrm{~ms}$ allowing for increased energy storage [19], and Ytterbium doped $K Y\left(W O_{4}\right)_{2}(\mathrm{Yb}: \mathrm{KYW})$ has a $1 \mathrm{~ms}$ lifetime but a quantum defect of as low as 3 percent allowing for more efficient pumping. The emission bandwidth of these materials is a few tens of nanometers depending on operating conditions, although Yb:KYW has a larger bandwidth and resulting reduced gain cross section [20]. 
Emission Properties of $\mathrm{Yb}^{3+}$ in Crystal. Hosts

( $\tau_{\mathrm{r} m}=$ measured emission lifetime: $\tau_{r u d}=$ lifetime calculated by reciprocity: $\tau_{s}=$ average or selected lifetime: $\Delta E=E_{Z I .}-h \nu ; \beta_{\min }=$ minimum fractional population inversion required to have transparency at the extraction wavelength; $\sigma_{c x t}=$ emission cross section at extraction wavelength judged to be optimal; parentheses suggest greater uncertainty in the magnitude of the quantity.)

\begin{tabular}{|c|c|c|c|c|c|c|c|}
\hline Host & $\begin{array}{l}\tau_{c m}(\mathrm{~ms}), \\
\text { Measured }\end{array}$ & $\begin{array}{c}\tau_{\text {rad }}(\mathrm{ms}) \\
\text { Calculated }\end{array}$ & $\begin{array}{c}\tau_{x}(\mathrm{~ms}) \\
\text { Selected }\end{array}$ & $\lambda_{e x r}(\mathrm{~nm})$ & $\Delta E\left(\mathrm{~cm}^{-1}\right)$ & $\beta_{\min }$ & $\sigma_{c x t}\left(10^{-20} \mathrm{~cm}^{2}\right)$ \\
\hline $\mathrm{LiYF}_{4}$ & 2.16 & 2.27 & 2.21 & 1020 & 480 & 0.098 & 0.81 \\
\hline $\mathrm{LaF}_{3}$ & 2.22 & 2.10 & 2.16 & 1009 & 353 & 0.113 & 0.36 \\
\hline $\mathrm{SrF}_{2}$ & 9.72 & $(8.6)$ & 9.2 & 1025 & 593 & 0.054 & 0.16 \\
\hline $\mathrm{BaF}_{2}$ & 8.20 & $(7.8)$ & 8.0 & 1024 & 578 & 0.058 & 0.14 \\
\hline $\mathrm{KCaF}_{3}$ & 2.7 & $(4.0)$ & 2.7 & 1031 & (593) & 0.070 & 0.22 \\
\hline $\mathrm{KY}_{3} \mathrm{~F}_{10}$ & 2.08 & 1.66 & 1.87 & 1011 & 369 & 0.118 & 0.44 \\
\hline $\mathrm{Rb}_{2} \mathrm{NaYF}_{6}$ & 10.84 & - & 10.84 & 1012 & $(372)$ & 0.140 & 0.10 \\
\hline $\mathrm{BaY}_{2} \mathrm{~F}_{8}$ & 2.04 & - & 2.04 & 1018 & $(458)$ & 0.097 & 0.67 \\
\hline $\mathrm{Y}_{2} \mathrm{SiO}_{5}$ & 1.04 & - & 1.04 & 1042 & $(617)$ & 0.047 & 0.33 \\
\hline $\mathrm{Y}_{3} \mathrm{Al}_{5} \mathrm{O}_{12}$ & 1.08 & 0.93 & 1.01 & 1031 & 628 & 0.055 & 2.03 \\
\hline $\mathrm{YAlO}_{3}$ & 0.72 & $0.42^{\mathrm{a}}$ & $0.42^{u}$ & 1014 & 353 & 0.141 & 1.31 \\
\hline $\mathrm{Ca}_{5}\left(\mathrm{PO}_{4}\right)_{3} \mathrm{~F}$ & 1.08 & 1.30 & 1.2 & 1043 & 603 & 0.046 & 5.90 \\
\hline $\mathrm{LuPO}_{4}$ & 0.83 & - & 0.83 & 1011 & 349 & 0.160 & 0.53 \\
\hline $\mathrm{LiYO}_{2}$ & 1.13 & - & 1.13 & 1020 & $(474)$ & 0.090 & 0.56 \\
\hline $\mathrm{ScBO}_{3}$ & 4.80 & - & 4.80 & 1022 & $(472)$ & 0.091 & 0.19 \\
\hline
\end{tabular}

${ }^{2}$ Only one of three polarizations accounted for.

Figure 1.6: Laser characteristics for a number of different hosts to the $Y b^{3+}$ ion (tungstate hosts not included) [19]

\subsubsection{Temperature Dependence of Yb:YAG Characteristics}

An advantage of using Yb:YAG over these other materials lies in the parameters related to high average power performance. Figure 1.7 shows a comparison of thermal conductivity traits for different materials which is a chief concern for high average power operation. Increased thermal conductivity allows for more efficient heat removal. The data in Fig. 1.7 shows that YAG in terms of heat conductivity is better than all of the other host materials tested for the $Y b^{3+}$ ion. It can also be noted from fig 1.7 that various material characteristics change as the material temperature is varied over a range of a few hundred Kelvin.

A number of favorable changes occur when Yb:YAG is cooled to cryogenic temperatures. As is evident from fig 1.7 the thermal conductivity experiences an increase by a factor of nearly ten when the temperature is decreased from room temperature to sub-100K temperatures. Other advantages of cryo-cooling the material include 


\section{Thermal Conductivity}
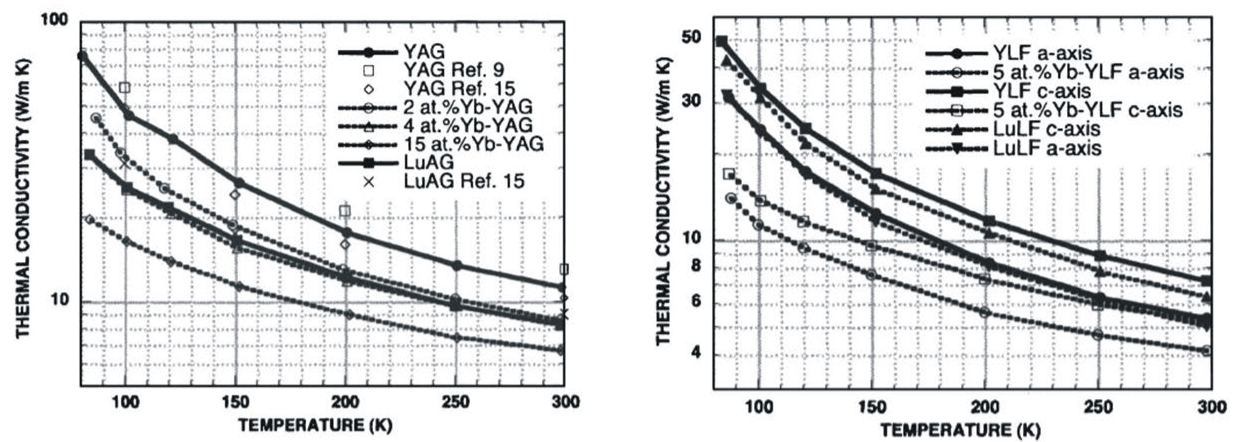

Thermo-optic

Coefficient
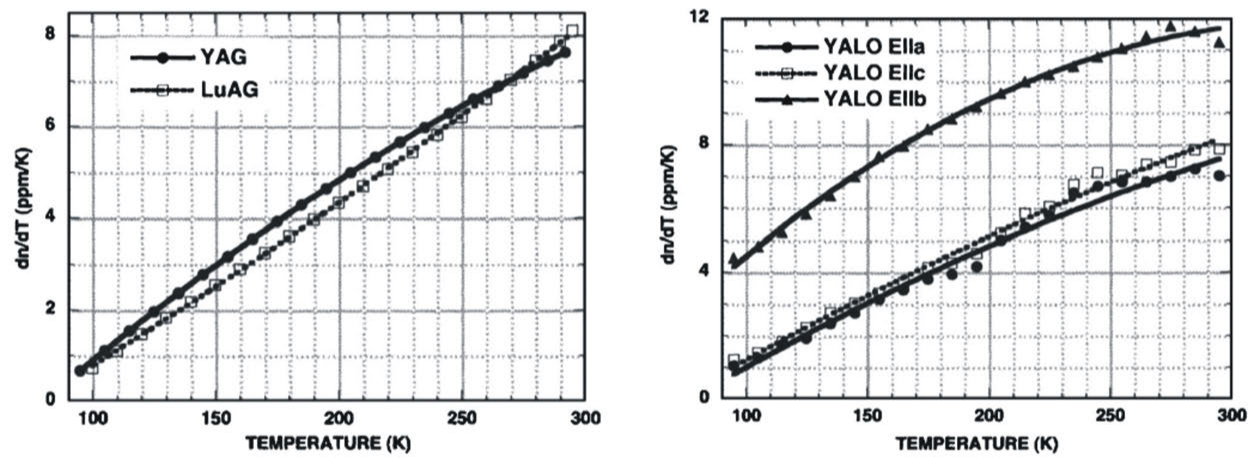

Coefficient of Thermal Expansion
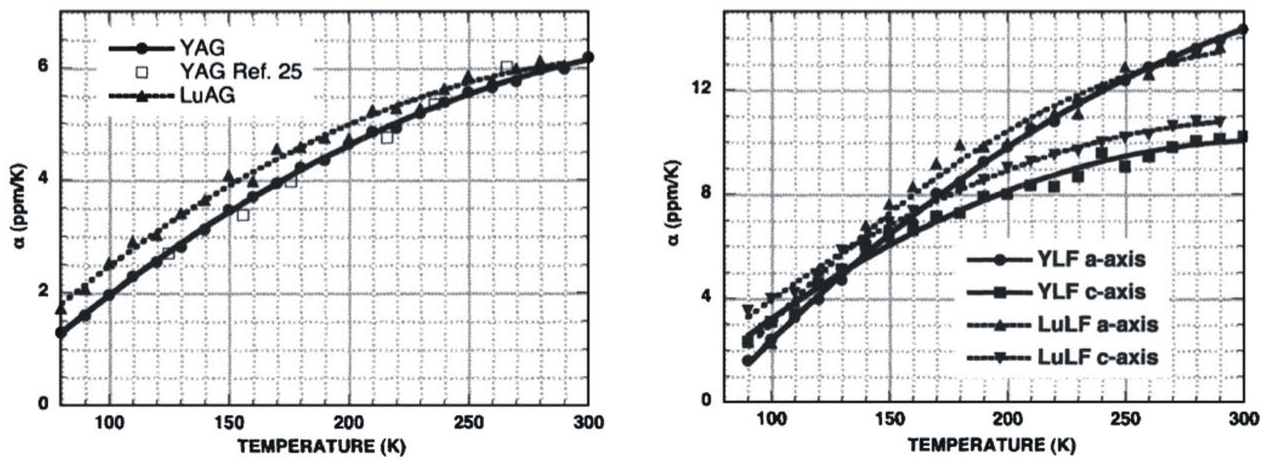

Figure 1.7: Thermal characteristics of YAG compared to other materials doped with $\mathrm{Yb}$ in the temperature range of $80-300 \mathrm{~K}$. It is important to note the different scales on some of the adjacent figures [18] 
a reduction by a factor of 4 in the thermal expansion coefficient which leads to less thermally induced mechanical stress. Any mechanical stress in the material will cause wavefront deterioration. Also the thermo-optic coefficient is decreases by a factor of around 7 , which also helps reduce thermal lensing in the case where there is a radial temperature gradient across the laser crystal [18]. A radial thermal gradient is a typical an almost unavoidable result of edge cooling of the lasing material [13].

Another temperature dependent property of $\mathrm{Yb}: \mathrm{YAG}$ is the initial lower laser level population. At room temperature the $Y b^{3+}$ ion has a quasi-three level energy structure. Aquasi-three level energy structure means that the lower laser level is not entirely depopulated in equilibrium. If the lower laser level has some population, than creating a population inversion is more difficult and requires more pump energy. If the lower level is depopulated in equilibrium and decays into the ground state, then the electronic structure is called a four level system. A four level system will be more efficient because less energy is necessary for a population inversion.

Based on the Boltzmann relation, the population of any two atomic energy levels, $N_{1}$, and, $N_{2}$, separated by an energy, $\triangle E$, at a temperature $\mathrm{T}$ is

$$
N_{2}=\left(g_{2} / g_{1}\right) N_{1} \exp (-\triangle E / k T)
$$

where $\mathrm{k}$ is Boltzmann's constant and $g_{2}$ and $g_{2}$ are the multiplicities of the levels. For Ytterbium, where the difference is $612 \mathrm{~cm}^{-1}$, about five percent of the ground state population inhabits the lower laser level at 300K. This increases the pump power necessary for inverting $\mathrm{Yb}$ at room temperature. This is a reason Neodymium has been used for many high power laser applications rather than Ytterbium, because it is a four level system at room temperature [15].

This quasi-three level behavior at room temperature is an additional reason to consider cryogenic conditions for $\mathrm{Yb}$ :YAG and is illustrated in Fig. 1.8. Cryogenic 


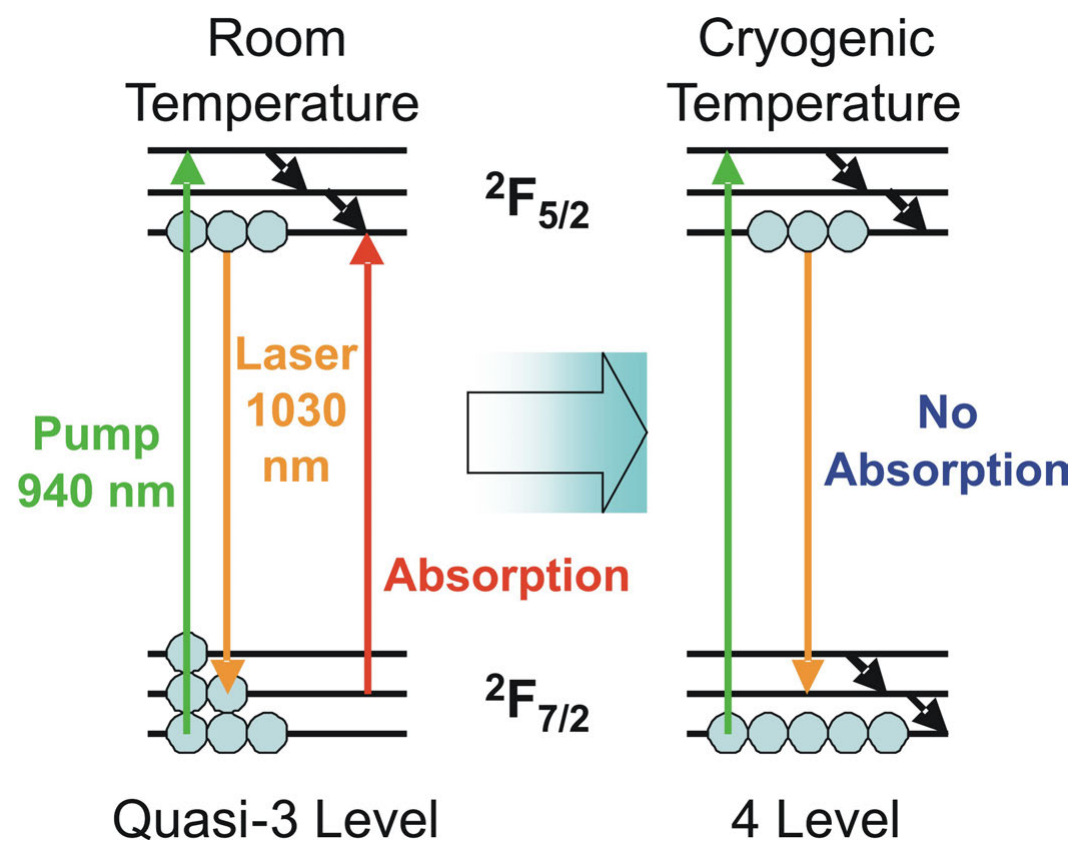

Figure 1.8: When Yb:YAG is cryogenically cooled it goes from having a quasi 3-level atomic energy structure to being a true 4-level system

cooling of the material causes the Yb:YAG lower energy level to be effectively depopulated, resulting in a true four level energy structure. This change allows for lower pump powers to reach threshold, as the material no longer needs to be 'bleached' to transparency by the pump light in order to create a population inversion.

The final, and perhaps most important to this work, Yb:YAG temperature trait is the emission bandwidth. Between cryogenic and room temperatures, the gain bandwidth changes by a factor of about 7, from around $10 \mathrm{~nm}$ to closer to $1 \mathrm{~nm}$, which is shown in fig 1.9. This has several important implications. Firstly by building laser amplifiers using the material at room temperature, one can maintain a much larger bandwidth. This means that a shorter transform limited pulse duration can be obtained. Depending on the applications the laser is to be used for, shorter pulse durations can be highly desirable.

Moreover, at cryogenic temperatures, due to bandwidth narrowing, the stimulated emission cross section of Yb:YAG dramatically increases (by a factor of about 7) which is shown in Fig. 1.10 [21].This will lead to higher gain per pass through the crystal in 


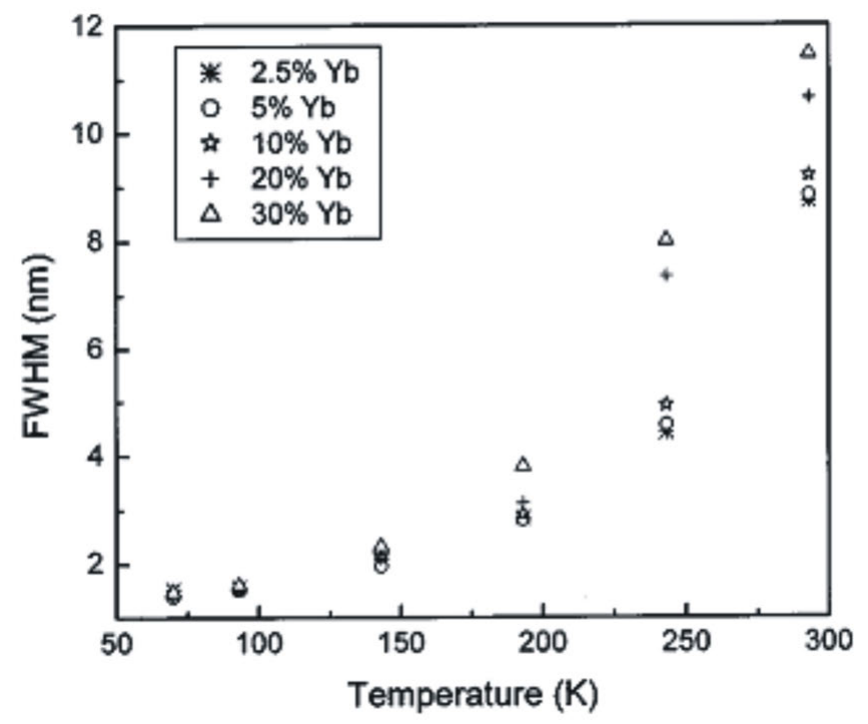

Figure 1.9: FWHM spectral line width for $\mathrm{Yb}: \mathrm{YAG}$ as a function of temperature and different doping concentrations [21].

an amplifier, resulting is fewer crystal passes, reduced wavefront degradation, reduced losses from other optics, and simpler amplifier geometry. This also means that the saturation fluence at cryogenic temperature is decreased by about the same factor which allows for much more efficient energy extraction. However, This will also result in a reduction in compressibility. Thus, a tradeoff is necessary between bandwidth and gain when one considers room temperature or cryogenic temperature operation.

In conclusion the $Y b^{3+}$ ion is considered as the lasant material due to the long upper level lifetime which allows it to be pumped by laser diodes. YAG is looked at as the host because it functions well under high average power operation. The excellent high average power properties of Yb:YAG can be further augmented by cooling the material to cryogenic temperatures, although this comes at the cost of a reduction of gain bandwidth. 


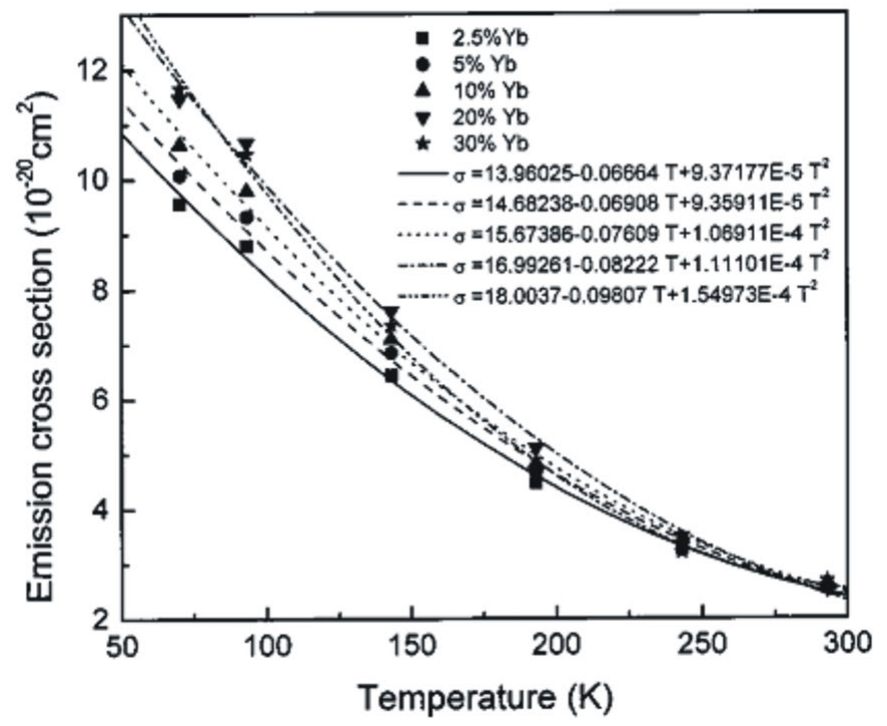

Figure 1.10: Stimulated emission cross section as a function of temperature for different doping concentrations for Yb:YAG [21]

\subsection{Cooling Geometry}

Another consideration in a solid state laser system is how cooling of the gain material is to be implemented. How this will be accomplished is foremost determined from the geometry of the gain media itself, i.e. whether it is a rod, or slab, or thin disk. For example, if one chooses to use lateral cooling of a rod, radial heat gradients cause a radial index of refraction gradient which will cause the material to act like a lens. This thermal lens effect becomes more exaggerated depending on how efficiently pump light is converted to laser light rather than heat and also depends on how efficiently heat can be removed from the material, which depends in turn on thermal conductivity. This usually only becomes a problem at higher pump powers in latter amplification stages.

One possible solution for reducing this problem is to use face cooling of the gain material. A common way such cooling is implemented is the active mirror configuration illustrated in Fig. 1.11. In this configuration, the active media is typically a thin disk with an anti-reflection coating on one of the faces and a reflection coating on 


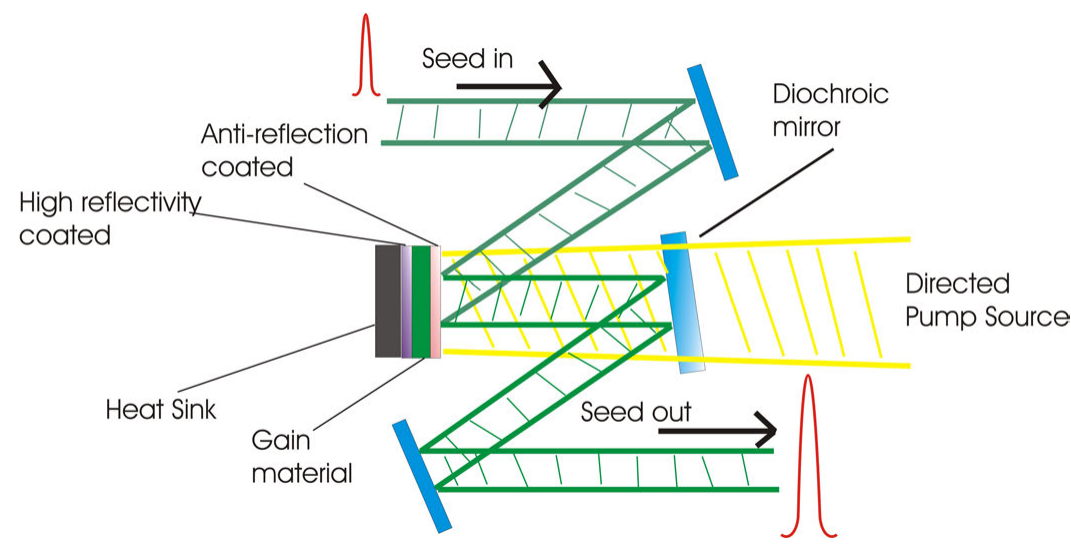

Figure 1.11: A typical active mirror amplifier configuration.

the other face. This allows the mirrored face of the gain media to be directly cooled while the crystal acts as a mirror in a cavity or amplifier. This cooling arrangement allows for maintaining higher beam quality [15]. Another advantage to the active mirror configuration is that per single pass through an amplifier, the gain media is double passed upon each reflection, resulting in higher gain per single pass through the cavity than a transmission based geometry.

\subsection{Diode-Pumped Yb:YAG CPA Systems}

Several all-diode-pumped Yb:YAG chirped pulse amplification laser systems have been developed recently. Some of these systems operate at room temperature $[22,23$, $24]$ and some are cryogenically cooled [25, 26, 27]. There are applications that are currently being investigated for these lasers. These are optical parametric chirped pulse amplification (OPCPA) [9] and soft x-ray laser generation [28]. In either case, diode pumping can allow for an increased repetition rate, resulting in a higher photon flux which is central to both applications. Further, if the CPA pulses can be amplified to higher energies, it is easier to actually enact these applications. These systems mostly use active mirror gain media configurations, because it is usually more effective for high repetition rate operation, for the above stated reasons. 
In an OPCPA a very broadband seed, which is also very compressible is amplified in a non-linear crystal that is simultaneously irradiated by a short duration high energy pump source where energy is transferred to the short seed pulse by parametric amplification. Because the amplification is parametric there is no energy storage in the crystal, which means that there are virtually no heat dissipation issues, but also means that the pump pulse duration must be on the order of the seed pulse duration. Additionally high pulse energies can be reached in a single pass through the parametric amplifier, making amplifier geometries simple. Less passes through the gain media reduces possible wavefront aberrations as well as possible B-integral issues [9]. Some very short duration, high power laser systems have been demonstrated in this way $[29,30]$, and plans to increase the pulse energy of these systems is underway.

Soft x-ray laser generation in the 10 to $20 \mathrm{~nm}$ spectral region can be accomplished by focusing a sequence of very high intensity, short duration laser pulses onto a metal target. the pulse produced by the CPA system are amplified to above the Joule level, and focused into a small line on the target creating a plasma column. The plasma can be created with the correct atomic conditions for extreme ultraviolet or soft x-ray amplification which in turn define the requirements of the pump pulse $[28,6]$. Previous and contemporary work in diode pumped $\mathrm{Yb}$ based CPA systems is discussed next.

\subsubsection{State of the Art Room Temperature CPA's}

The first room temperature system that will be mentioned was developed by J Tummler et. al. at the Max Born institute in 2009[22]. The oscillator is a diode-pumped Yb:KGW followed by two Yb:YAG thin disk diode pumped amplification stages. 165 milliJoules of energy per pulse are reported at $100 \mathrm{~Hz}$. After the first stage the pulses can be compressed to under 2 picoseconds. The intended application for this laser is high average power x-ray laser generation, which is why amplification to the 
Joule level is repeatedly mentioned as a project goal. To date, no room temperature Yb:YAG CPA system has gotten much closer to the Joule level than this.

A room temperature Yb:YAG system which is intended for use as a pump laser for a parametric amplifier was developed in 2009 by Thomas Metzger et. al. [23]. This system produces $25 \mathrm{~mJ}$ of pulse energy with a single regenerative amplifier, at up to $3 \mathrm{kHz}$. The seed for the amplifier is generated by a Ti:Sapphire system and has more bandwidth than the seed mentioned in the previous system. After being amplified to $25 \mathrm{~mJ}$, close to $1 \mathrm{~nm}$ of bandwidth remains which allows the pulses to be compressed to $1.6 \mathrm{ps}$.

The final room temperature system that will be mentioned was developed by Klingebiel et. al. in 2011 [24]. The pulses are generated in a Ti:sapphire mode-locked oscillator and proceed through a two $\mathrm{Yb}^{3+}$ amplifier system, a regenerative Yb:glass amplifier and a Yb:YAG multipass amplifier. The entire system runs at $10 \mathrm{~Hz}$ and is able to produce $200 \mathrm{~mJ}$ pulses which compress to $1 \mathrm{ps}$, maintaining $3.5 \mathrm{~nm}$ of bandwidth. This is once again a system that is intended for OPCPA applications. Unlike the other two room temperature systems discussed above, this CPA does not take advantage of thin disk technology, which is one of the reasons that it is limited to $10 \mathrm{~Hz}$ at a similar energy to the first mentioned system.

\subsubsection{State of the Art Cryogenic Temperature CPA's}

The first cryogenic system of interest to this work was published in 2007 by Akahane et. al. [25]. The system, developed for OPCPA pumping once again, has a Ti:Sapphire oscillator producing pulses which are coupled into a cryo-cooled Yb:YAG regenerative amplifier which boosts the pulse energy to $8 \mathrm{~mJ}$ at $10 \mathrm{~Hz}$. The repetition rate was limited by the diode driver capabilities. The system output had a 35 ps pulse duration. A quasi thin disk amplifier arrangement is used in the amplifier (1 mm thick crystal), which does not necessarily limit the repetition rate, but has different consideration 
which must be taken into account for amplifier design such as doping and cooling than true thin disk structures. Although this CPA clearly produces less energy than some of the previously mentioned systems, at a lower repetition rate, it must be considered that it was the first of the aforementioned systems developed.

Another cryogenic CPA system developed in 2010 for OPCPA application was developed by Hong et. al. [26]. This system is a two amplification stage cryo-cooled Yb:YAG system which produces $40 \mathrm{~mJ}$ pulses at $2 \mathrm{kHz}$. After the first stage amplifier $6.2 \mathrm{~mJ}$ pulses are achieved at $15 \mathrm{ps}$ duration after compression. This is once again not a thin disk system, but is still able to operate at a high repetition rate due to the cryogenic temperature operating conditions. Comparing this system to the similar system [23] above, it can be noted that higher energy pulses which are longer after compression at a similar repetition rate are achieved with the cryogenic system as we expect.

The last example of a cryo-cooled Yb:YAG all-diode-pumped CPA that will be addressed before looking at the main subject matter of this work is intended for soft x-ray generation. This system is actually the predecessor to the CPA that will be discussed in detail below, and was published in 2009 by Furch et. al [27]. The CPA consists of a Yb:KYW mode-locked oscillator and two cryo-cooled amplification stages. The first stage is a regenerative amplifier which works at up to $100 \mathrm{~Hz}$ and produces around $8 \mathrm{~mJ}$ energy per pulse. After this first stage the spectral bandwidth has been reduced to close to two angstroms. The second stage is a twelve pass amplifier which boosts the pulse energy up to $1.4 \mathrm{~J}$ but only at up to $10 \mathrm{~Hz}$ due to gain material heating, with minimal further gain narrowing. The pulses finally were compressed to 8.5 ps with 1 Joule of energy.

Because the system to be subsequently discussed is based on an overhaul of the system just mentioned, and is an attempt to improve beam quality, compressibility, and repetition rate, some further discussion is warranted. In order to further compress 
the amplified pulses, bandwidth must be better maintained. There are a number of possible approaches to this problem which include trying to seed the amplifiers with much broader bandwidth pulses, using a gain material that has a broader emission bandwidth, or changing the operating temperature of the amplifiers in order preserve more of the bandwidth of the oscillator seed. Ultimately both the seeding bandwidth and the operating temperature were changed in order to help compensate the gain narrowing caused by the cryogenic Yb:YAG.

Increasing the repetition rate and beam quality leaving the second stage amplifier are problems that are still to some degree under development as far as the scope of this work goes. At this point the repetition rate coming from the second stage amplifier can easily reach $100 \mathrm{~Hz}$ but with a resultant output pulse energy of 100mJ. Additionally, trouble with the pulse profile itself originated with the high pump powers on the crystal and an inefficient cooling scheme to deal with the heating of the Yb:YAG. More consideration will be given to these matters in the conclusion section of this report entitled 'future work'.

\section{6 conclusion}

To conclude the introduction to this work, a number of concepts which are directly important to the system have been reviewed. Solid state lasers have been talked about in detail. Different types of gain material and relating considerations when building an oscillator or amplifier have been discussed, particularly where the $Y b^{3+}$ ion and $Y b^{3+}$ doped YAG are used as the gain material. Pulsed lasers were briefly mentioned, although there is a great deal more that can be talked about where a mode-locked oscillator is concerned. Diode pumped chirped pulse amplification systems have been discussed in detail with a number of specific examples of state of the art systems, many of which are similar in a number of ways to the system that will be discussed 
in detail following. 


\section{Chapter 2}

\section{System Overview}

\section{$2.1 \quad$ Overview}

This chapter discusses the Yb:YAG all-diode-pumped chirped pulse amplification laser system that was partially developed as part of this thesis. The laser system consists of a mode-locked oscillator, and two stages of amplification. The pulses generated at $56 \mathrm{MHz}$ by the $\mathrm{Yb}: \mathrm{KYW}$ oscillator are centered around $1030 \mathrm{~nm}$ spectrally. Pulses exiting the oscillator have about ten nanoJoules of energy. The pulses are directed into a folded dielectric Offner grating stretcher, where the pulse duration is increased from 200 fs to 400 ps. Due to poor grating efficiency the pulses are reduced to picoJoules of energy after passing through the stretcher. The pulses are coupled into the first Yb:YAG amplification stage where they are amplified up to the milliJoule level. The pulses which had been exiting the oscillator at a $56 \mathrm{MHz}$ repetition rate are allowed into the amplifier at a rate of $100 \mathrm{~Hz}$ during typical operation. The pulses next proceed to the second Yb:YAG amplification stage where they are further amplified to $140 \mathrm{~mJ}$. Finally the pulses are sent to a dielectric grating compressor where the duration is reduced to 5 picoseconds with a pulse energy of $100 \mathrm{~mJ}$. A diagram of the system is presented below. 


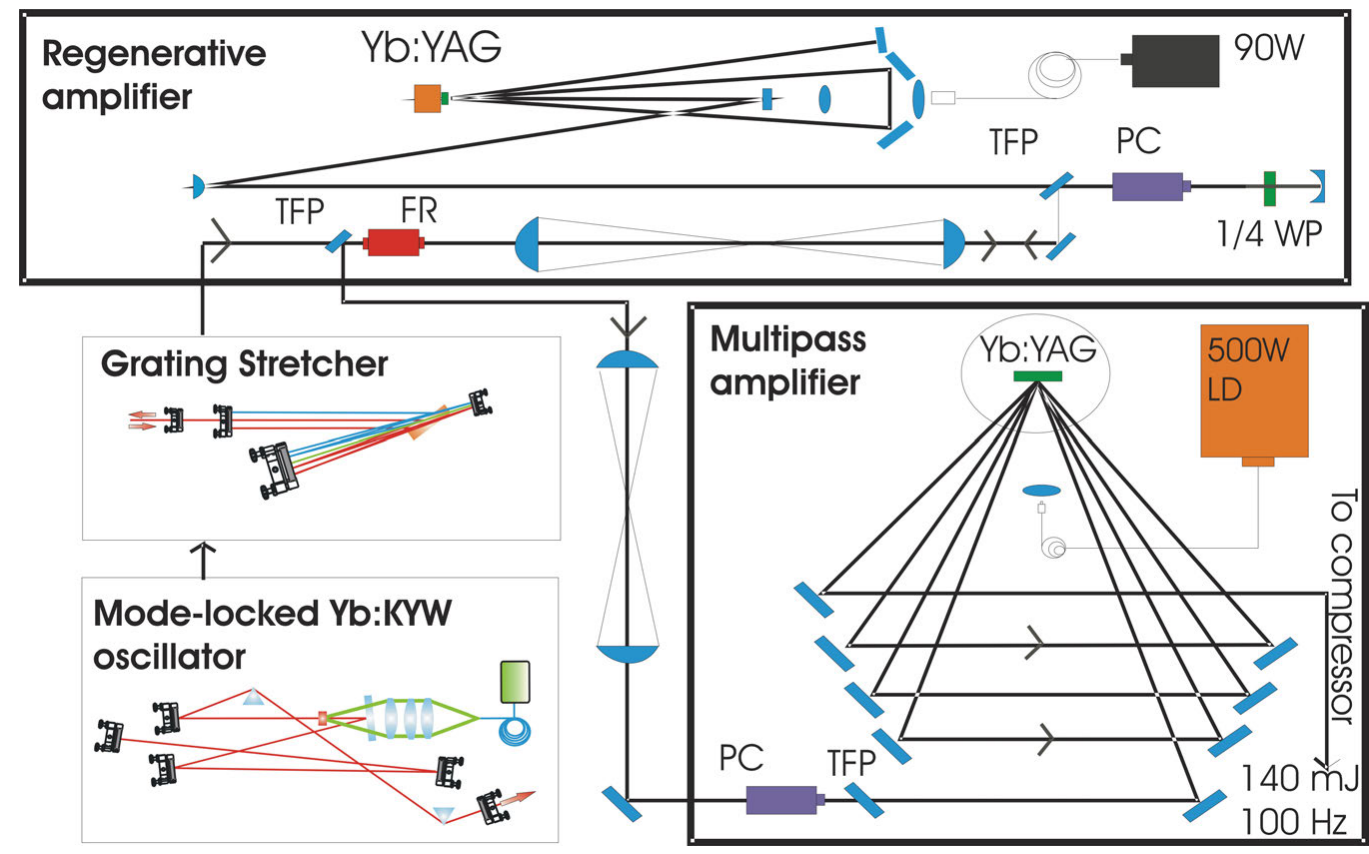

Figure 2.1: Schematic diagram of the compact, diode-pumped Yb:YAG laser system. TFP, thin film polarizer; FR, Faraday rotator; PC, Pockels cell; WP, waveplate; LD, Laser diode.

As the laser pulses pass through the system there are a number of parameters that change notably. One such parameter is the spectral bandwidth of the pulses. As the pulses pass through each amplifier, some amount of bandwidth is lost due to gain narrowing in the gain media. This effect is exacerbated by cooling the laser crystals to cryogenic temperatures in the second stage. The bandwidth narrowing is important to monitor because, as was mentioned above, less bandwidth causes the transform limited pulse duration to increase.

The cryo-cooling of the second stage is a result of another important consideration in the system. As the pulses are amplified in each stage, increased pump energy must be deposited into the gain media. As a result thermal effects become more problematic. As was previously discussed, cryo-cooling of Yb:YAG can significantly aid in the reduction of such deleterious effects, as well as increase amplifier efficiency. Finally, and also related to the heating issues mentioned above, the wavefront of the laser pulses must remain fairly uniform throughout amplification if the laser is to be 
suitable for application.

\subsection{Semi-Conductor Laser Diode Pumps}

All of the semi-conductor laser diodes used to pump the system are InGaAs based and are commercially available. Both amplifiers are pumped at $940 \mathrm{~nm}$, a broad absorption band for Yb:YAG. The oscillator, which uses a Yb:KYW crystal for the gain media is pumped at $980 \mathrm{~nm}$, the optimum wavelength for KYW. The oscillator is also pumped continuous wave (CW) as opposed to the amplification stages which are pumped quasi-continuous wave (QCW) as necessary for stable mode-locking. Due to the upper level lifetime of $\mathrm{Yb}$ :YAG which is approximately one millisecond, pumping the crystals with one to two millisecond QCW pulses is fairly efficient, with the stored energy efficiency increasing with shorter pump pulse durations. Data showing different output energies at 3 different pump durations can be seen in the regenerative amplifier section below.

The laser diodes emittance wavelengths can be tuned by changing the operating temperature. This is important because although the diodes can be designed to emit at specific wavelengths for a particular temperature, they are not always manufactured on that mark. For this reason the diode cooling water supply must be controlled to keep them operating at the optimum temperature. Due to the fact that the diodes tend to emit at slightly shorter wavelengths than $940 \mathrm{~nm}$ by a few nanometers, typically the diodes have to be heated to some degree to shift the spectrum to the correct wavelength. Figure 2.2 shows a shift of $5 \mathrm{~nm}$ in wavelength of the 2 nd stage amplifier diode corresponding to a 10 degree Celsius change in temperature. One unfortunate side effect of increasing the diode temperature is that the threshold current for lasing increases, thus decreasing wall plug efficiency. Also the necessary increase in current and operating temperature decreases the useful diode lifetime. 


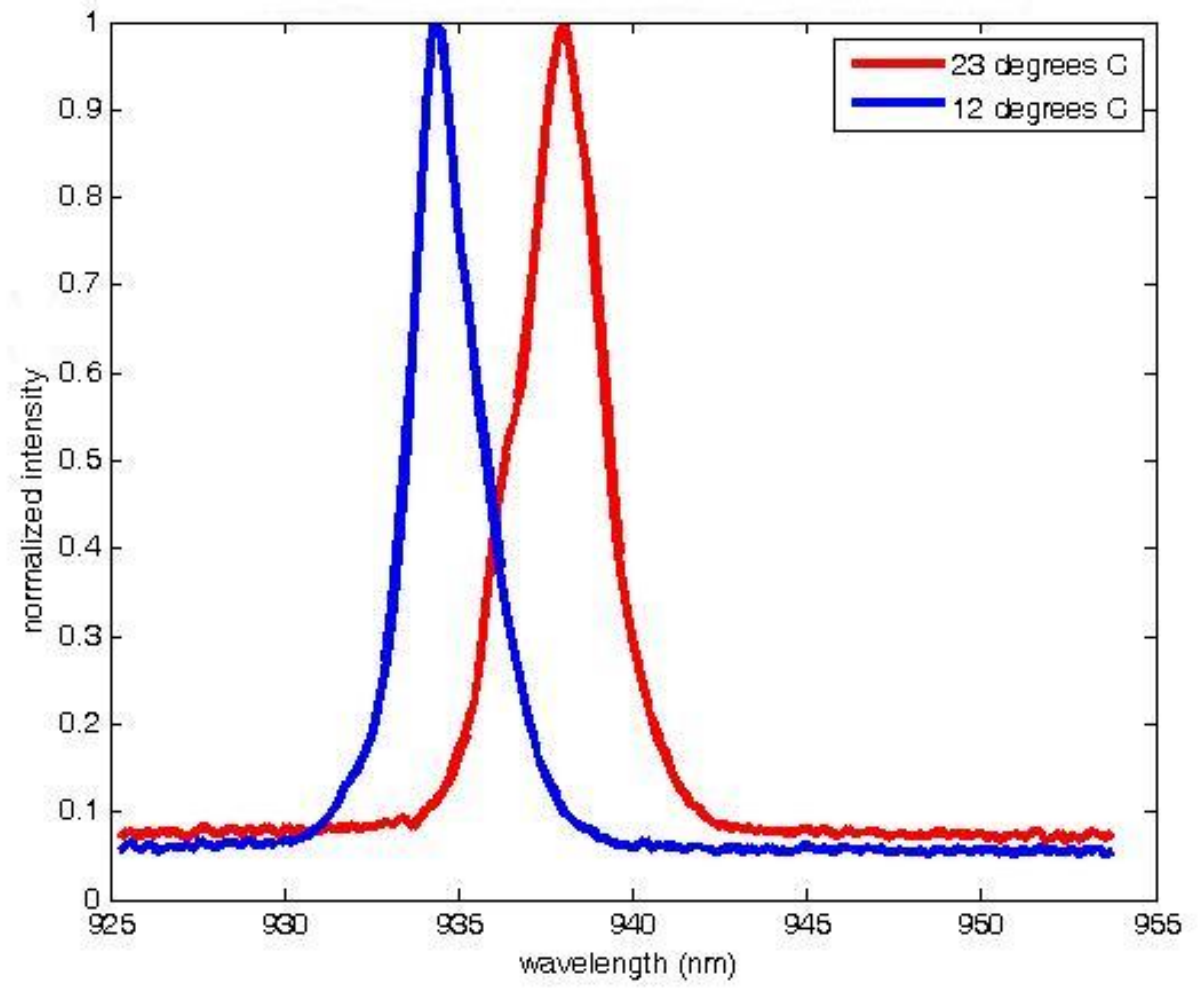

Figure 2.2: Spectra of the diode used to pump the second stage amplifier. A significant center wavelength shift of several nanometer is evident with an operating temperature adjustment of only 10 degrees. 
There are a number of different pumping geometries used throughout the system to deposit the energy in the gain media without interfering with the beam path. In the oscillator, the pump is focused into a 200 um spot on the crystal using a 2 inch concave gold mirror to mode-match the cavity as closely as possible. A small hole is drilled into the center of the mirror to allow the beam to pass through. The fiber which couples the laser diode light from the diode enclosure to the crystal imaging system is positioned to minimizes astigmatism by keeping the incident angle on the pump mirror as small as possible. The diode itself puts out $30 \mathrm{~W}$ maximum of average optical power. However the oscillator pump is run at closer to $20 \mathrm{~W}$ to prevent instabilities in the cavity such as double pulsing.

The regenerative amplifier uses a dichroic mirror to pump the cavity crystal along with a lens based imaging system. The light generated by the $90 \mathrm{~W}$ peak power diode laser exits the fiber with a half angle of 15 degrees and is ultimately imaged into a $700 \mathrm{um}$ spot on the crystal which is quite close to mode matched to the cavity which is discussed later. The dichroic mirror is over 95 percent transmissive at the 940 nm pump wavelength and over 99 percent reflective at the $1030 \mathrm{~nm}$ seed wavelength. Although a similar geometry to the oscillator pump system could possibly be more efficient here due to losses in the lenses and dichroic mirror, testing this method revealed a good deal of astigmatism. The astigmatism was caused by the crystal mount geometry necessitating a larger incident angle on the concave pump mirror. The aberated pump led to poor beam quality of the amplified seed at the output of the amplifier.

The second stage amplifier has the simplest pumping geometry. The $500 \mathrm{~W}$ peak operating power diode is coupled to a fiber that is imaged using a $35 \mathrm{~mm}$ focal length lens on the crystal. The image size has a $4 \mathrm{~mm}$ diameter. Because there is no dichroic mirror for the stage, care must be taken to make sure that the seed does not become incident on the imaging lens for the pump during alignment. If the amplified seed 
were to be imaged by the lens back into the fiber it could travel back down the fiber to the diode stack itself resulting in possible diode damage or fiber damage due to the high peak intensities. The laser diodes are one of the more costly components of the system.

\subsection{Gain Media}

The Yb:YAG and Yb:KYW crystals in the various sub-sections of the system are mounted in different ways to account for different conditions. In order to effectively cool the crystals, good thermal contact has to be made between the crystals and the heat exchangers for each crystal. In both the oscillator and the two amplifiers the crystal is thermally contacted to copper using indium, where the copper itself is either water cooled or liquid nitrogen cooled. The mounts must additionally be engineered so that they do not put mechanical stress on the crystal which can lead to distortion of the wavefront or even crystal fracture. This stress can be the result of a heating or cooling process when the crystal is rigidly attached to a mount which has a different thermal expansion coefficient than the crystal material.

The doping level of the gain media in each stage is a separate consideration. In all three stages the dopage of the crystals is determined by the available pump power foremost. In the case of the oscillator the Yb:KYW crystal should be able to store a large fraction of the pump energy, but still experience a population inversion to enable lasing. In the amplifiers that follow, The pump must still create a population inversion. However, the gain-length product is also a consideration, where higher doping should lead to the same gain in a thinner crystal, at the same pump power. Also higher doping in a room temperature crystal will increasingly populate the lower laser level, which is not true at cryogenic temperatures. The doping level of the amplifier crystals can also cause slight shifts in the gain center bandwidth which can 
reduce overall gain of the system if there is a mismatch between stages.

In the oscillator the crystal is a 5 atomic percent doped Yb:KYW with $5 \mathrm{~mm}$ by $5 \mathrm{~mm}$ by $2 \mathrm{~mm}$ dimensions. It is mounted on a water cooled copper heat sink. The mount itself consists of a small ring which presses the crystal into the bulk of the mount which has a chiller water line attached to it for temperature control. The mount is made for transmission operation with the beam passing through the crystal at normal incidence, thus the crystal has a dielectric multilayer anti-reflection coating on both faces. The thermal lens created by pumping with the $30 \mathrm{~W}$ diode is taken into account when calculating cavity stability. The cavity analysis is shown further below.

The regenerative amplifier Yb:YAG crystal is a 10 atomic percent doped $10 \mathrm{~mm}$ in diameter $1 \mathrm{~mm}$ thick crystal. Rather than being mechanically pressed to the heat sink and mount this crystal is soldered using indium. The mount is again cooled by a chiller for temperature control. In order to create a good bond between the indium and the crystal and thus good thermal contact, three thin layers of chromium, nickel, and gold are evaporatively deposited on the back of the crystal. This means that one side of the crystal is coated for high reflectivity and one side for transmission of the $1030 \mathrm{~nm}$ light.

The second stage amplifier crystal is a $30 \mathrm{~mm}$ by $30 \mathrm{~mm}$ by $5.5 \mathrm{~mm}$ with 2 atomic percent doping. This crystal is also in an active mirror configuration. This crystal is soldered using the same thin metal coatings on the reflection face and indium soldered to a copper heat sink. However in this case the copper heat sink is cooled with liquid nitrogen and hangs in a vacuum chamber. The seed beam and pump light must pass through an anti-reflection coated window into the vacuum jacket to reach the crystal. The nitrogen dewer holds about 4.5 liters of liquid $N_{2}$ when full which can keep the crystal cooled while the pump is in regular operation at $100 \mathrm{~Hz}$ for about 8 to 10 hours. Warping of the beam front is not a problem due to crystal strain as the crystal 
is cycled through an 80-300 $\mathrm{K}$ temperature range.

Both the second and third stage crystals fall into a thick disk catagory. The basis of thin disk technology is that a crystal which is several hundred micron thick is cooled in an active mirror configuration. This allows for very fast axial heat removal for high pump powers, and thus higher repetition rates can be achieved. In this scheme the doping must be very high to obtain significant gain. This in turn leads to a very high gain-length ratio in the transverse direction if the beam is more than a few millimeter

in diameter. The transverse amplified spontaneous emission (ASE) can significantly reduce the stored energy and be a limiting factor for amplification. By using a thick disk, longitudinal gain can be maintained and transverse gain can be reduced by reducing the doping which reduces transverse ASE. There is some tradeoff here where the cooling of gain media becomes less effective, which will ultimately reduce the possible repetition rate.

\subsection{Isolation Between Stages}

The oscillator and each of the amplification stages are separated by isolation optics to keep spontaneous emission from feeding forward or backward in the system. This is accomplished by controlling the polarization of the light as it moves through a Pockels cell surrounded by a pair of polarizers. Two crossed polarizers keep light from passing through as long as a Pockels cell between them does not effect the polarization. If, however, a voltage is applied to the anisotropic crystal in the Pockels cell it will rotate the polarization. In the case of linearly polarized light, if a certain voltage is applied, the polarization can be rotated from $\mathrm{S}$ to $\mathrm{P}$ or vice-versa. This is known as a half wave voltage on a Pockels cell. Thus only when this switching voltage is applied will light pass unattenuated through allowing its use as an optical isolator. As long as the switching is done sufficiently fast, it is possible to propegate only the pulse of interest 
but reject the passing of any other light, effectively isolating the stages.

A slightly different method of isolation is used for the output of the oscillator. For this purpose a combination of a Faraday rotator and two crossed polarizers are implemented directly after the oscillator output coupler. The crossed polarizers once again keep any light from passing through except that the faraday rotator changes linearly polarized light from $\mathrm{S}$ to $\mathrm{P}$ or vice--versa allowing light of the correct linear polarization to pass through unattenuated. The Faraday rotator only operates in one direction allowing light leaving the oscillator to pass, while blocking any backward traveling pulses from reaching the oscillator. This is important because any feedback into a mode-locked cavity would interrupt the mode-locking process and disrupt short pulse generation. 


\section{Chapter 3}

\section{Oscillator and Stretcher}

\subsection{Oscillator}

The laser pulses are generated in a solid state mode-locked oscillator shown schematically in Fig. 3.1. The gain media is a Yb:KYW (ytterbium doped potassium gadolinium tungstate) which is mounted in a water cooled copper heat sink. Mode-locking is accomplished using a semi-conductor saturable absorber mirror (SESAM). As is evident from Fig. 3.1, the SESAM is one of the end mirrors of the cavity, and the other end mirror is the output coupler. A multilayer dielectric chirped mirror is used to compensate intercavity dispersion and facilitate short pulse generation. The crystal is pumped by a continuous wave $30 \mathrm{~W}$ fiber coupled InGaAs laser diode. The total round trip cavity length is close to five meters, giving a pulse repetition rate of 56 MHz. The pulses contain close to $20 \mathrm{~nJ}$ of energy, giving a total output power of close to one Watt. The pulse duration was measured using second order autocorrelation. The pulse was determined to be close to be 265 fs at the full width half maximum pulse duration assuming a $\operatorname{sech}^{2}$ fit.

The results of a ray trace analysis for the cavity are shown in Fig. 3.2. The ray trace is done assuming the beam has a Gaussian transverse profile, and the lines 


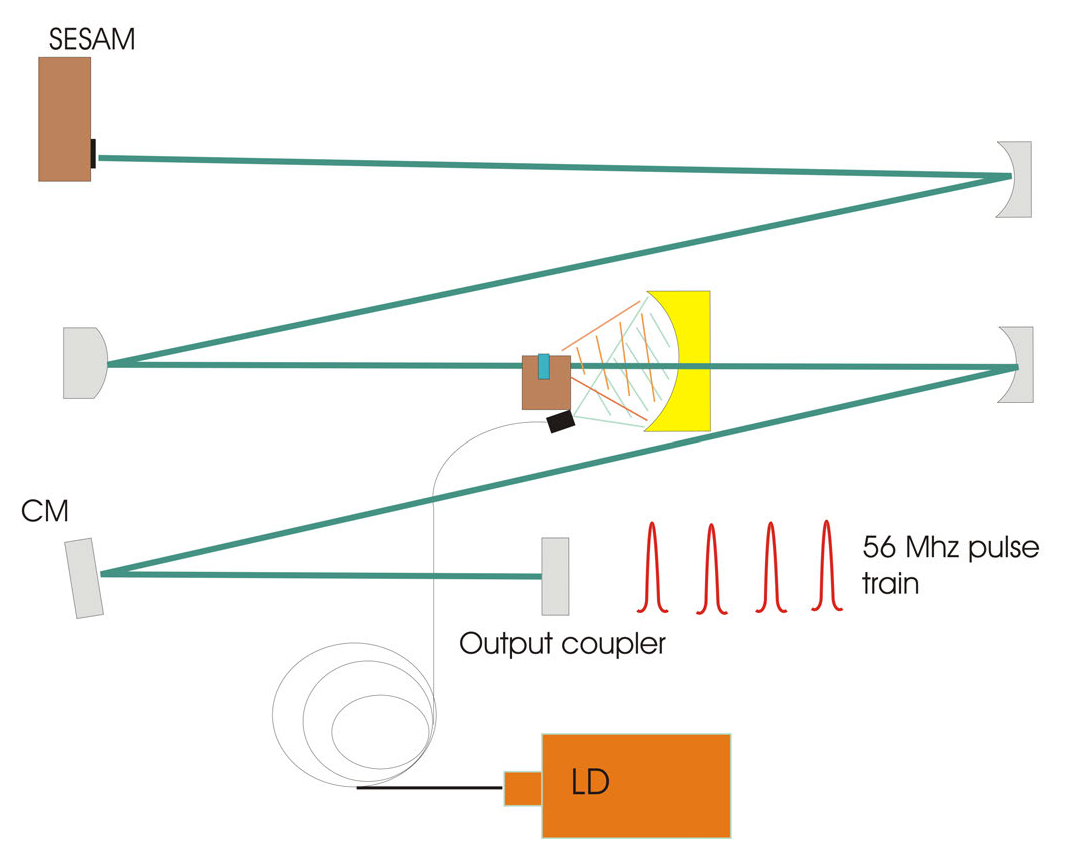

Figure 3.1: Schematic diagram of the Yb:KYW mode-locked oscillator. LD, Laser diode; CP chirped mirror; SESAM, semi-conductor saturable absorber mirror.

indicate the 2 omega spatial extent of the electric field (wherein 67 percent of the electric field amplitude is located in the case of a Gaussian). One of the waists is located on the saturable absorber end mirror where high intensities are necessary for the saturable absorber to enable mode-locking conditions. Another waist is located on the Yb:KYW crystal where higher pump intensities allow for higher small signal gain. The almost collimated section of the beam is sent to the other end mirror which is also the output coupler.

The basic layout of the SESAM used to mode-lock the cavity is shown schematically in Fig. 3.3. By simply absorbing noise level energy, a pulse will be preferentially amplified as was discussed above in the mode-locking section. There are a number of parameters that can be controlled when these mirrors are in production. The saturable absorption, for example, is important because it determines a threshold for what electric field amplitude will be damped out of oscillation, thus giving a minimum pulse intensity threshold for laser action. Higher absorption results in increased threshold pump power for the cavity also. A related parameter is the modulation 


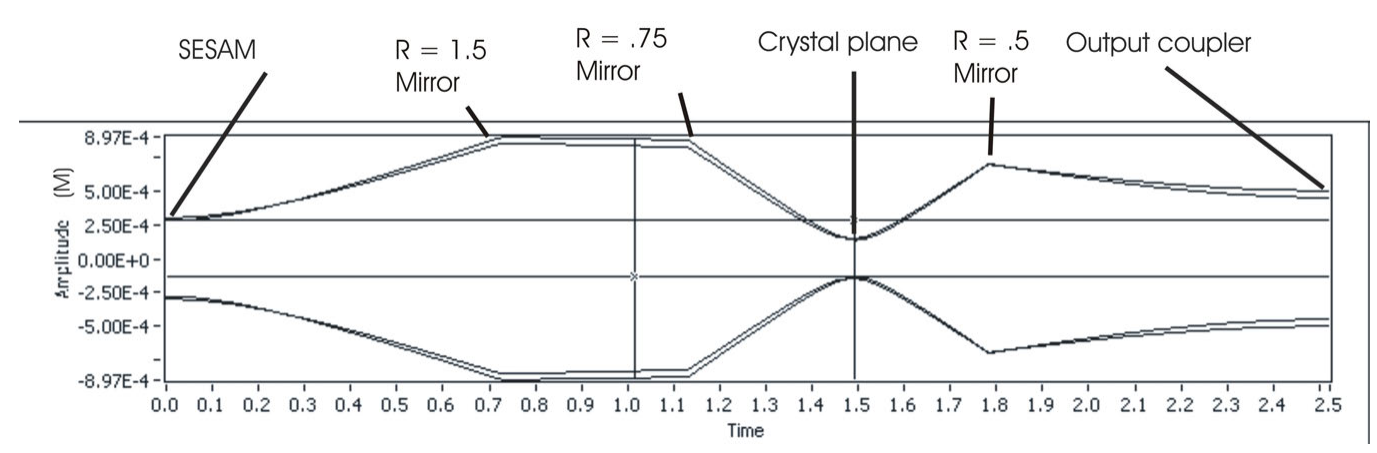

Figure 3.2: Raytrace of oscillator cavity. The amplitude shown in meters is the electric field amplitude and so field intensity is found from dividing this value by the square root of 2 . The lower axis is in time but can be converted to space by dividing by the speed of light.

depth of the SESAM which takes into account non-saturable absorption as well as saturable absorption. Modulation depth is typically different from the saturable absorption by some amount due to gain media imperfections that lead to absorption in active material. Saturation fluence determines the size of the cavity mode on the SESAM. Finally the reflection and absorption bandwidth will determine how short of a pulse the cavity can support if these values are less than the gain material emission bandwidth [31].

In a mode-locked cavity some type of dispersion compensation is necessary to maintain short pulse durations. A chirped mirror is used for this purpose in the oscillator. A chirped mirror is a dielectric stack of alternating high and low refractive indices, much like any dielectric stack optical mirror, except that different wavelengths penetrate to different depths, thus effectively taking different path lengths before being reflected. This is similar to the prism dispersion compensation schemes discussed in the mode locking section, except that dispersion happens only longitudinally and not transversly as is the case with prism dispersion compensation. Thus if one of these mirrors is designed correctly, the natural positive dispersion of a mode-locked cavity can be compensated per round trip. Additionally, one mirror simplifies alignment and increases the stability of the cavity as opposed to two or four prisms. 


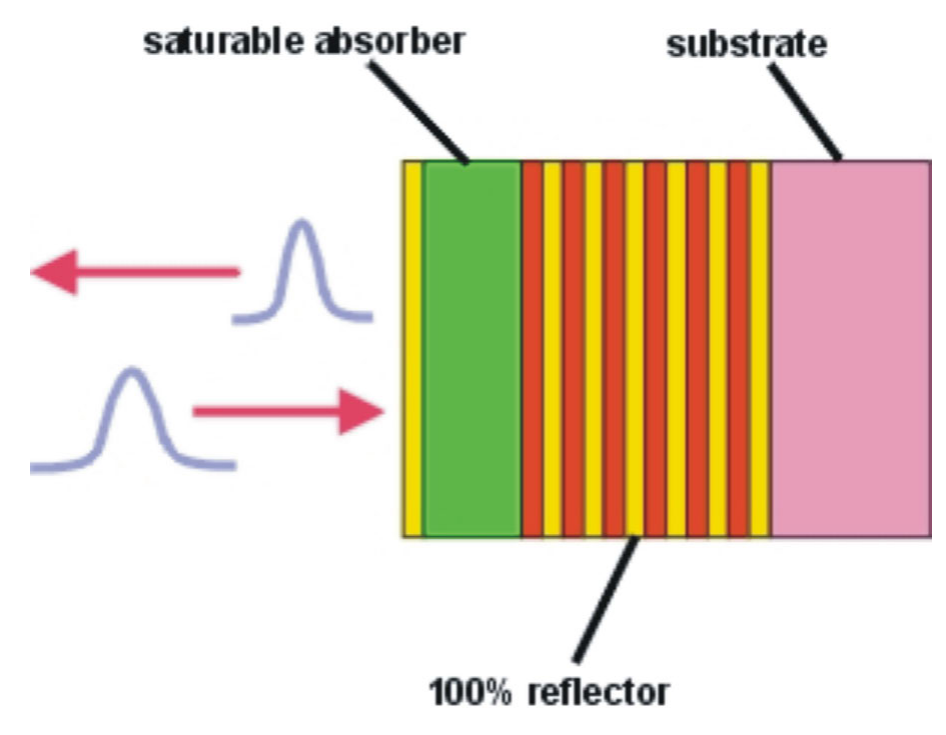

Figure 3.3: A semi conductor saturable absorber mirror schematic from the company Batop. A top layer quantum well is where the saturable absorbtion occurs is deposited on a high reflectivity dielectric stack coating [31].

The oscillator chirped mirror is based on the design from Ref [32]. In Fig. 3.4 below, the dispersion characteristics are shown for the chirped mirror in the oscillator cavity where it can be seen that a $1030 \mathrm{~nm}$ pulse is imparted with $-2000 f s^{2}$ per mirror reflection. This group velocity dispersion (GVD) value is based on calculated dispersion caused by the crystal. The fact that the pulse duration is at the transform limit for the bandwidth of the oscillator, which is discussed below, indicates that the mirror negative GVD matches the crystal positive GVD quite well.

The output spectrum of the oscillator can be seen in Fig. 3.5. The spectrum is shifted to the red side of the optimum Yb:YAG $1030 \mathrm{~nm}$ wavelength, but Yb:KYW is a broader band gain medium than Yb:YAG, which makes short pulse generation easier. Using Yb:KYW as the gain media for the oscillator also means that the rest of the system can be seeded with more bandwidth. The oscillator has some amount of frequency tunability depending on crystal temperatures. Allowing the crystal to increase in temperature, by changing the cooling water temperature, shifts the center of the spectrum to higher wavelengths, and broadens the spectrum by up to a few 


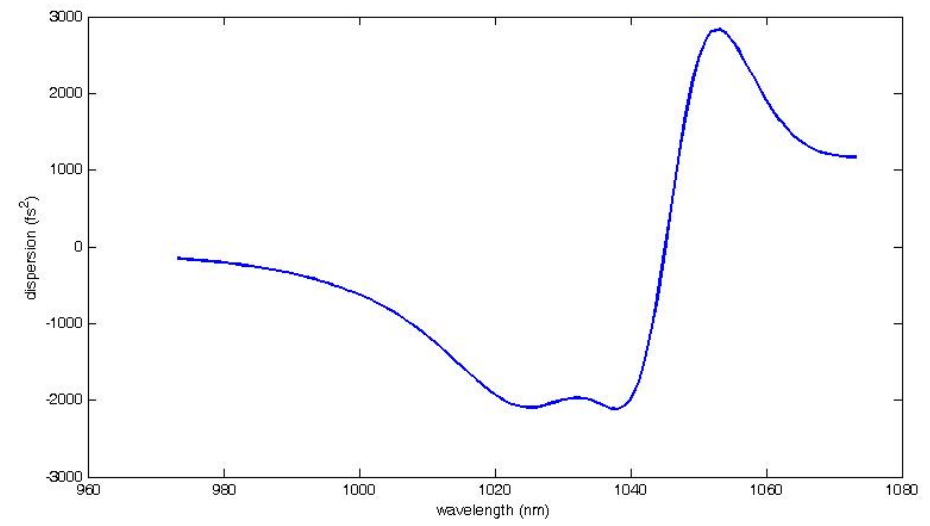

Figure 3.4: Theoretical dispersion curve for design of chirped mirror in oscillator cavity.
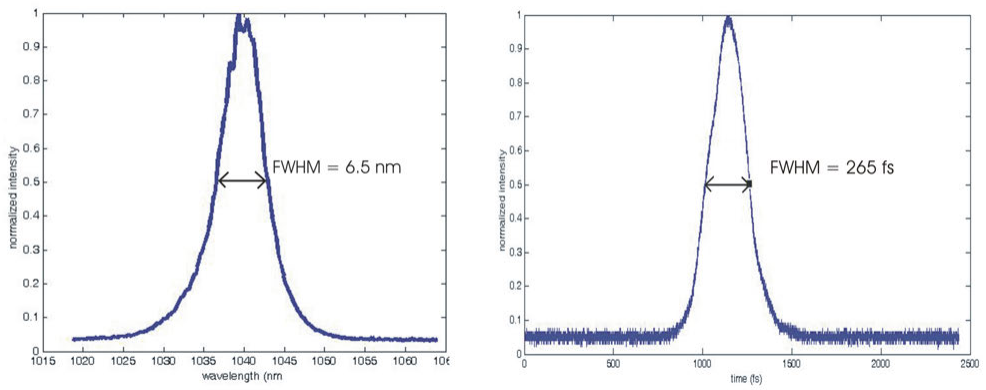

Figure 3.5: left: Spectrum of mode-locked laser oscillator. The cavity is operated at room temperature. right: Autocorrelation of the oscillator pulses without deconvolution factor. Using a sech ${ }^{2}$ deconvolution factor (dividing by 1.54) we arrive at a pulse duration of 265 fs.

nanometers.

The temporal duration of the pulses exiting the oscillator are measured by second order autocorrelation. This is a technique where the pulse is split and reoverlapped in a non-linear crystal giving a frequency doubled intensity cross-correlation of the pulse duration. The resulting oscillator pulse duration is 265 fs at the full width half maximum and the trace is shown in Fig. 3.5. Using the formula for the Fourier limit for the pulse duration $\triangle t$ based on the frequency bandwidth $\Delta \nu$ for an assumed $s^{2} c h^{2}$ pulse shape 


$$
\triangle t \triangle \nu=.36
$$

it can be noted the these are the shortest possible pulses that can be supported by the pulse bandwidth.

\subsection{Stretcher}

The stretcher takes the 265 fs pulses and stretches them in time to around 400 ps after gain narrowing. This is achieved by a double pass through the stretcher. A blazed holographic 1714 lines per millimeter gold grating is used to separate out the spectral components. The efficiency of the grating is about thirty percent resulting in an efficiency of only around one percent after the beam has been diffracted by the grating 4 times. Thus, a few tens of nanoJoules per pulse from the oscillator enter the stretcher and only a few hundreds of picoJoules come out.

A schematic of the stretcher used in the system can be seen below in Fig. 3.6 . The beam first impinges on a grating, and propegates through an imaging system composed of an Offner triplet. The lenses image the first grating to a position behind the second grating, effectively causing the distance between the two gratings to be negative much like the Martinez stretcher discussed above. In the case of an Offner stretcher however, the ratio of the radiuses of curvature of the two mirrors which is 2:-1, allows for the spherical and astigmatic abberations to cancel eachother from each mirror. Additionally no chromatic aberations occur because the light does not propegate through lenses.

Using the same formula from Treacy [12] for the second order spectral dispersion $\varphi_{2}(\lambda)$ for a grating separation $\mathrm{L}$ 


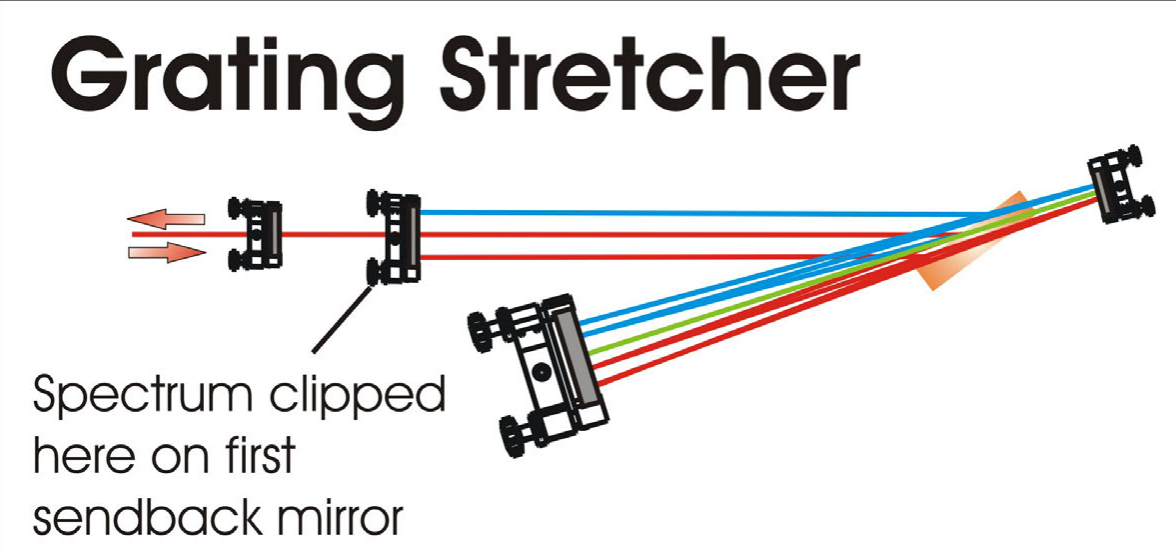

Figure 3.6: Schematic diagram of the Offner grating stretcher. The Offner configuration allows for increased time delay with less propagation distance as compared with the traditional Martinez configuration.

$$
\varphi_{2}(\lambda)=\frac{L(\lambda / d)}{c d\left[1-(\lambda / d-\sin \gamma)^{2}\right]}
$$

or because $\varphi_{2}(\lambda)=\frac{\Delta T}{\Delta \lambda}$ where $\triangle T$ is the temporal stretch imparted to the pulse per unit bandwidth, $\triangle \lambda$

$$
\triangle T=\frac{L(\lambda / d) \triangle \lambda}{c d\left[1-(\lambda / d-\sin \gamma)^{2}\right]}
$$

where $\mathrm{d}$ is the line density of the diffraction grating, $\mathrm{c}$ is the speed of light, and $\gamma$ is the incident angle on the grating.

Then for $L_{i}$, the distance of the image from the second grating we have

$$
L_{i}=4(L-f)
$$

for the Martinez stretcher and 


$$
L_{i}=4(L-2 f)
$$

for the Offner stretcher. It can be seen that the Offner configuration allows for extra stretch with less actual propegation in addition to having good abberation qualities.

Thus, for

$$
\begin{gathered}
\lambda=1030 \mathrm{~nm} \\
\triangle \lambda=.5 \mathrm{~nm} \\
d=1720 \text { lines } / \mathrm{mm} \\
\gamma=62^{\circ} \\
L_{i}=-4.8 \mathrm{~m}
\end{gathered}
$$

then

$$
\triangle T=200 p s
$$

for each pass through the stretcher.

The first bandpass filter that the pulses encounter when propagating through the system is in the stretcher. As the spectrum is dispersed through the stretcher, the beam expands horizontally. By the time the pulse arrives at the first send back mirror it is at least fifty percent bigger than the mirror. The spatial chirp of the beam causes the bandwidth to be filtered upon reflection. Close to 1.5 nanometers of bandwidth clear the stretcher. Although the first sendback mirror could be replaced by an optic 
that would clear more bandwidth, the narrow emission bandwidth of the subsequent amplifiers make further increasing the stretcher bandwidth a non-issue. 


\section{Chapter 4}

\section{Regenerative Amplifier}

\subsection{Description of a Regenerative Amplifier}

The term regenerative amplifier refers to a multipass amplifier that is also in a cavity in the case of optical amplifiers. The cavity is much like any other laser resonator, where issues of stability, alignment, and gain have to be taken into account. The only difference is that seed pulses must be coupled into the cavity, and back out once the energy has been extracted. This is as opposed to a typical resonator oscillator where the pulse oscillates indefinitely and some percentage of the total energy leaks from the output coupler every round trip.

While in the cavity the pulses will follow the natural transverse cavity modes. The pump pulse deposits the energy right before the pulse is coupled into the cavity to prevent the stable cavity from lasing on its own. For a laser diode pump source this can be fairly easily achieved if the right current source for the laser diode is available. If pumped appropriately, a regenerative amplifier should be able to act independently as a Q-switched cavity if no seed is injected. Indeed if the energy is not appropriately extracted by the seed (usually due to a timing problem), Q-switching in the cavity can result which not only reduces available amplification energy but also can disrupt 
the amplification process in the next stage.

\subsection{Description of Cavity and Optics}

The seed pulses are coupled into the regenerative amplifier by controlling the polarization of the light. This is done in a similar manner and with the same types of optics that are used to provide isolation between the stages. As shown in Fig. 4.1 The pulses enter the cavity by reflecting off of a thin film polarizer (TFP) while they are $\mathrm{S}$ polarized. The pulses then travel immediately through a Pockels cell (PC) followed by a quarter wave plate (WP), encounter one end mirror and are sent back on the same line they came in on. As long as the Pockels cell is off, the pulses are switched from $\mathrm{S}$ to $\mathrm{P}$ polarization after double passing the quarter wave plate allowing them to travel through the rest of the cavity. No other elements in the cavity effect the polarization, so the pulses are ejected from the cavity again after double passing the quarter wave plate the second time. If, however, the Pockels cell is switched to quarter-wave voltage while a pulse is in the cavity and to the left of the polarizer, when the in-cavity pulse double passes the combination of Pockels cell and polarizer, there will be no net effect on the polarization and the pulse will continue to make round trips through the cavity until the Pockels cell is switched off again. Additionally, any other pulses which enter the cavity while the Pockels cell is at quarter wave voltage, will be have a net zero polarization rotation, and will be coupled back out of the cavity without passing through the gain material and thereby depleting the gain.

As can be seen from Fig. 4.1 the 10 atomic percent active mirror Yb:YAG is double passed per single pass trip in the cavity, corresponding to a quadruple pass through the gain region. There are a number of reasons for using this geometry despite that fact that it makes alignment more difficult. The original intention was to negate possible spatial hole burning effects in the cavity [33] that would distort the 


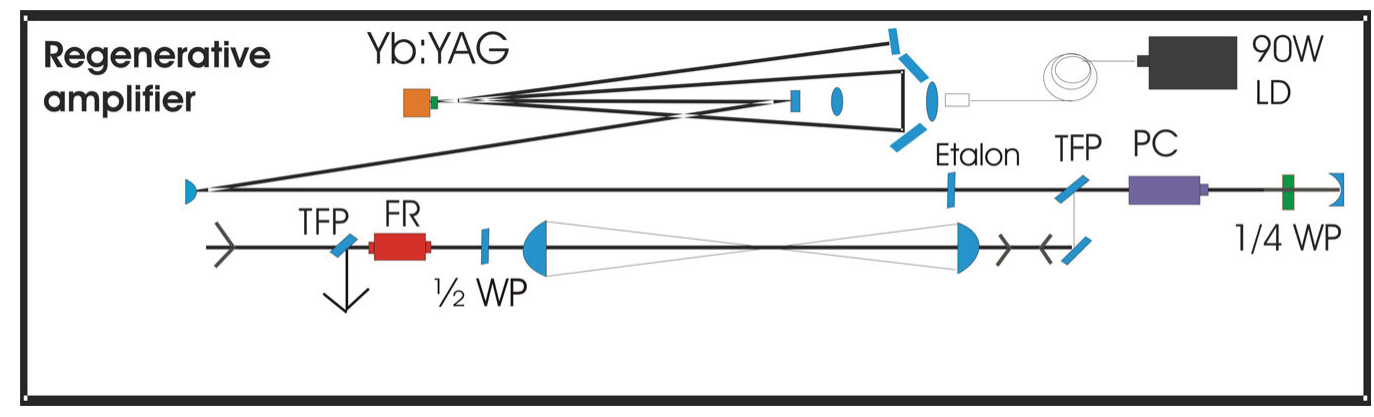

Figure 4.1: Schematic diagram of the first stage room temperature regenerative amplifier. TFP, thin film polarizer; FR, Faraday rotator; PC, Pockels cell; WP, waveplate.

spectrum of the output pulses. Additionally, the pulses reach saturation sooner due to the fact that the pulse experiences twice the amplification per single pass through the cavity while still seeing only single pass losses on the Pockels cell and polarizer. Thus, energy can be more efficiently extracted. Finally, output pulse energies from the amplifier are noticeably more stable when double passing the gain, most likely do to more rapid saturation that lends to reduced propagation.

Pulses are ejected from the cavity after an optimum number of passes. Pulse amplification is monitored by a leak through one of the end mirrors by a photodiode and oscilloscope. Examples of the signal from the photodiode can be seen in Fig. 4.2. Figure 4.2 shows cases of an undersaturated pulse, a well saturated pulse, and a pulse that has been left in the cavity too long and the losses have overcome the gain. By carefully controlling the number of round trips the pulse makes in the cavity, maximum energy extraction can be achieved. The optimum time of cavity containment changes depending on pump power, seed pulse energy, and cavity losses (how well the cavity is aligned). Typically for a well aligned cavity and full pump power, the pulses are kept in the cavity for 14 round trips.

Once the amplified pulses have been switched out of the regenerative amplifier, they are sent back on the same path that the seed beam came in and thus must be separated to avoid re-insertion into the stretcher and oscillator. This is accomplished by a Faraday rotator, a half wave plate, and a thin film polarizer. On the way into 

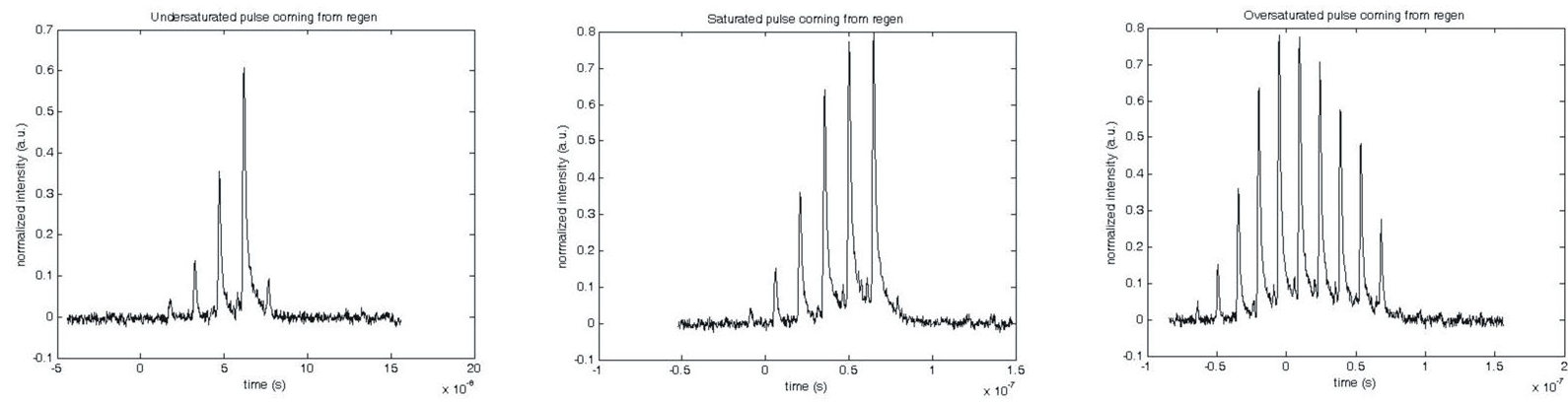

Figure 4.2: A leak through one of the end mirrors in the regenerative amplifier is monitered with a photodiode. How well saturated the gain in the regenerative amplifier is depends on how many round trips the pulse makes in the cavity. Here the cases of undersaturated, well saturated and oversaturated can be seen.

the cavity, the pulses pass through the polarizer as P-polarized light, are rotated to $\mathrm{S}$ by the half waveplate, are unaffected in passing through the Faraday rotator to be coupled into the amplifier by the cavity polarizer. After exiting the cavity as Spolarized light, the polarization is rotated to $\mathrm{P}$ by the Faraday rotator, and back to $\mathrm{S}$ by the half wave plate allowing them to be reflected by the first polarizer rather than moving back toward the stretcher which can all be traced in Fig. 4.1. This sends the pulses in the direction of the second stage amplifier.

Figure 4.3 shows a ray trace of the regenerative amplifier cavity assuming a Gaussian beam. The gain media is located in the almost collimated section of the beam on the right side of the figure. The crystal plane being marked twice indicates the gain media being double passed. The beam diameter is around 700 um where it passes through the crystal, where the almost flattop transverse pump has close to a $700 \mathrm{um}$ diameter also. The two curved mirrors indicated in the diagra and which are evident where the beam diameter starts changing more rapidly in the figure allow for cavity stability. The input-output coupler is located in the region where the beam diameter is changing, causing the beam to be slightly convergent upon being switched out of the cavity. A one-to-one telescope is placed directly after the output to collimate the beam. 


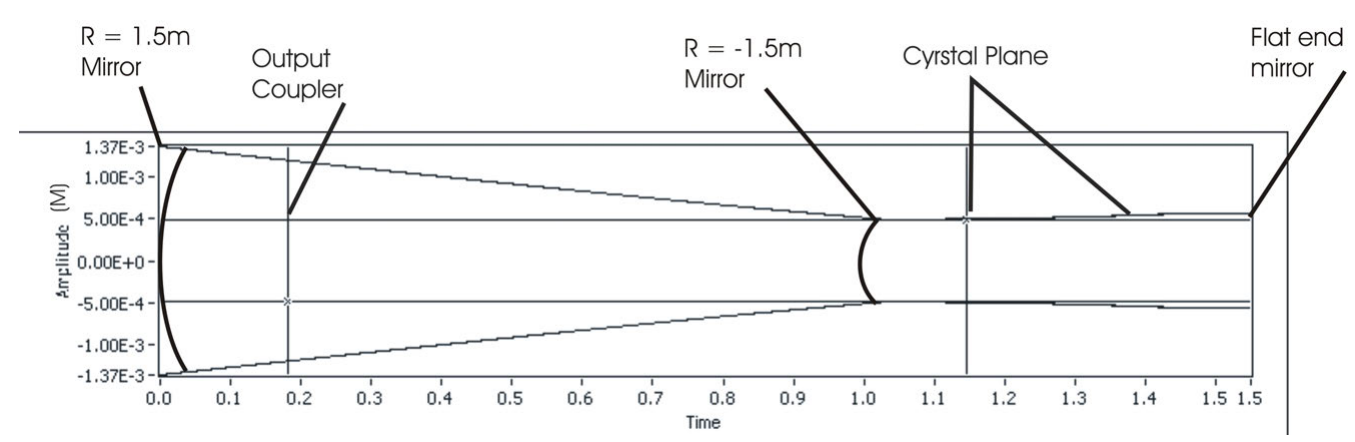

Figure 4.3: Raytrace of the regenerative amplifier cavity.

The cavity center wavelength can be tuned to some degree by an inter-cavity etalon. The etalon is designed to pass the $1030 \mathrm{~nm}$ wavelength of the seed at close to Brewsters angle, and allows for wavelength tunability of the cavity with very small angle variation. Fig 4.4 shows the transmission spectrum of the etalon for several different incidence angles. The spectral width of these peaks is larger than the gain bandwidth of the active media for this amplification stage, and thus upon amplification, is not a limiting factor in that respect. However, by choosing the placement of the peak wavelength, the cavity will preferentially amplify centered at that wavelength. The etalon itself consists of a pair of dielectric coatings on a substrate, separated by a layer of silicone dioxide which is thick with respect to the dielectric stacks. The thick layer acts like the etalon cavity and the reflectivity of either face is controlled by the characteristics of the two thin film coatings. Wavelength tuning of this amplifier is necessary to match the wavelength of this stage to that of the next amplifier which is slightly shifted due to different temperature and doping concentration.

\subsection{Regenerative Amplifier Results}

The intended characteristics of the regenerative amplifier are $100 \mathrm{~Hz}$ operation, with over a milliJoule of pulse energy and at least 3 angstroms of bandwidth while maintaining a good beam profile. Because of the large amplification in this stage (pico- 


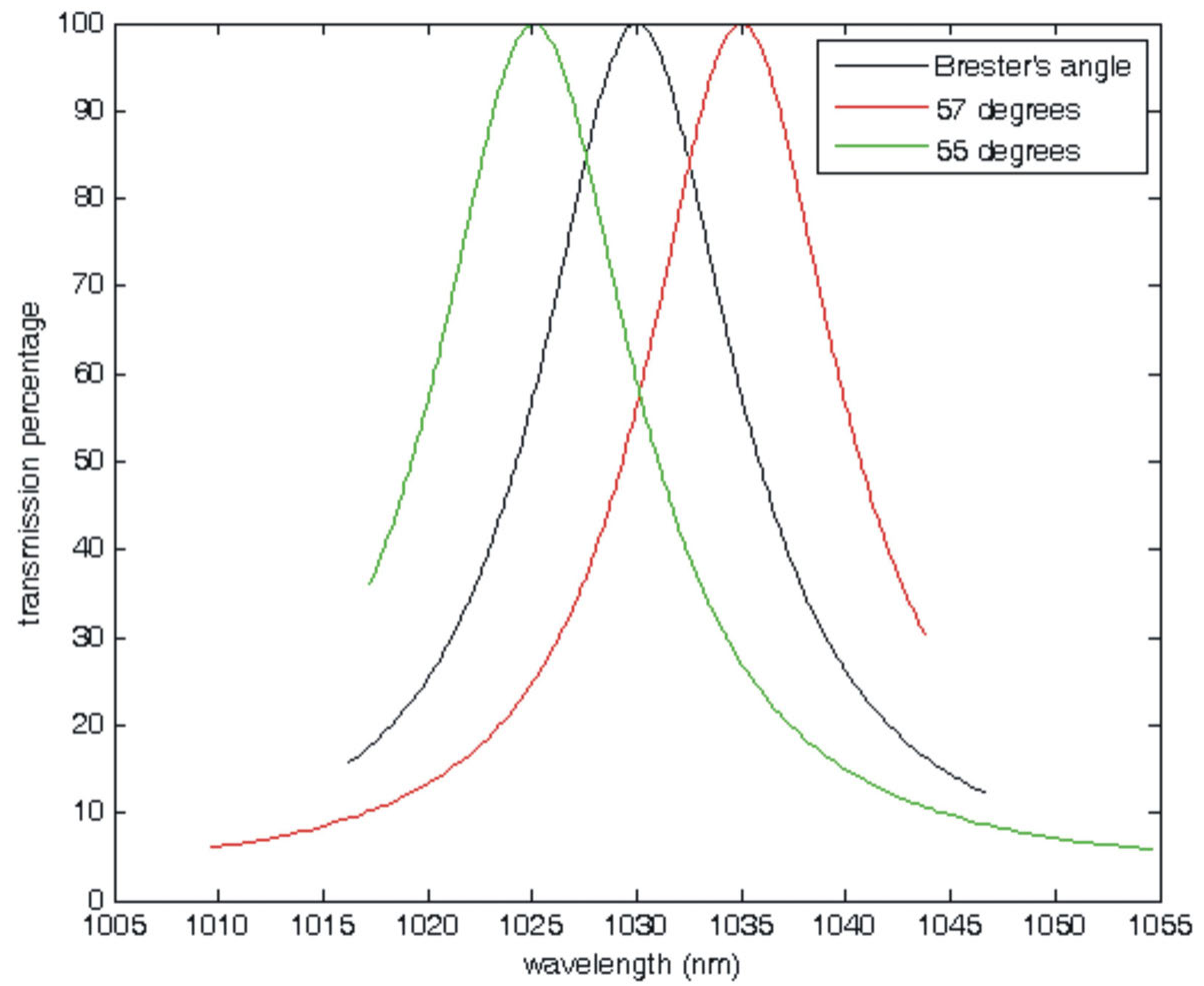

Figure 4.4: Etalon transmission spectrum for three different angles near Brewsters angle. Very small changes in incidence angle result in very large change in center bandpass wavelength. 
Joules to milliJoules) maintaining enough bandwidth to compress to sub-five picosecond pulse duration is essential. For the pulse to be further amplified and still be focusable, a good wavefront quality must be maintained through the amplification process.

The primary effect of increasing the repetition rate is heating of the gain media. In the case of this amplifier, this heating has negligible thermal lensing or gain reducing effects at up to $100 \mathrm{~Hz}$, but does cause small shifts in the cavity alignment. Thus, minor cavity re-adjustments are necessary as the repetition rate is increased to optimize output energy. The data shown in Fig. 4.5 is taken at essentially full pump power for the $90 \mathrm{~W}$ regenerative amplifier diode. The pump pulses are $84.5 \mathrm{~W}$ peak power with a 2 ms duration. The output energy starts to decrease at repetition rates greater than $100 \mathrm{~Hz}$. Also, as the repetition rate increases over $100 \mathrm{~Hz}$ the pump diode show signs of declining pump pulse peak power, which may contribute to the drop in amplified pulse energy.

Figure 4.6 shows a plot of different amplifier output energies as a function of different laser diode currents at $100 \mathrm{~Hz}$ repetition rate for different pump pulse durations. The maximum operating current for the regenerative amplifier pump diode is $50 \mathrm{~A}$, corresponding to about a 97 Watt peak power quasi-cw pulse. As can be seen from Fig. 4.6, the output energy can easily be extended into the several milliJoule range under several different pump pulse durations. Typically the amplifier is operated with a $2 \mathrm{~ms}$ pump pulse duration, producing about $3 \mathrm{~mJ}$ of energy. After beam shaping and isolation optics, this allows $2 \mathrm{~mJ}$ of energy to reach the next amplification stage. Increasing the pump duration beyond 2 ms does not significantly increase the pulse energy.

The regenerative amplifier shows good stability over a range of pumping conditions. Figure 4.7 shows the standard deviations of the output energy from the amplifier for the same pumping conditions that are shown in figure 4.6. Although 


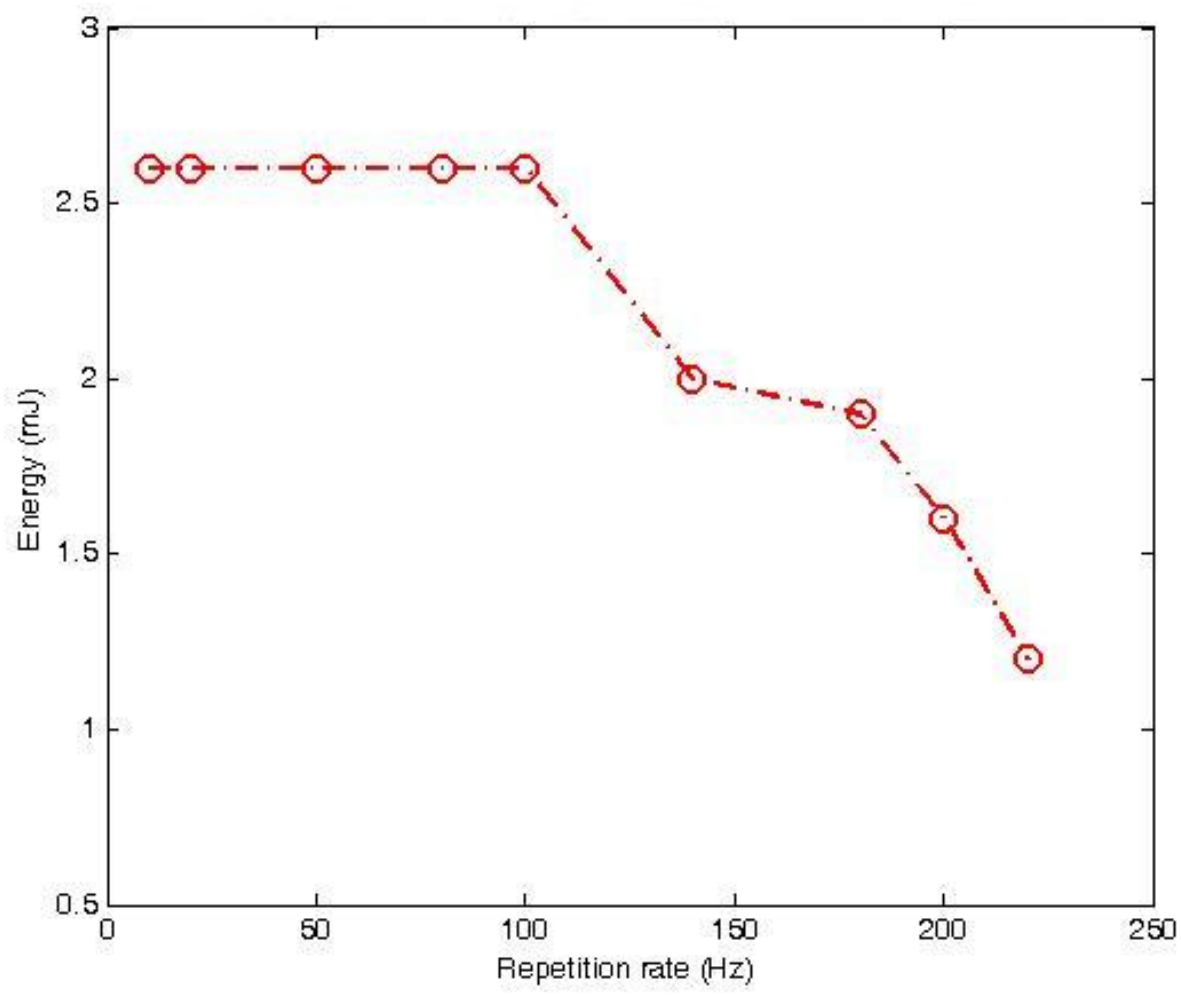

Figure 4.5: Regenerative amplifier output pulse energy as a function of amplifier repetition rate. The regenerative amplifier shows no energy degradation up through $100 \mathrm{~Hz}$ and can be run at higher repetition rates with some loss of energy.

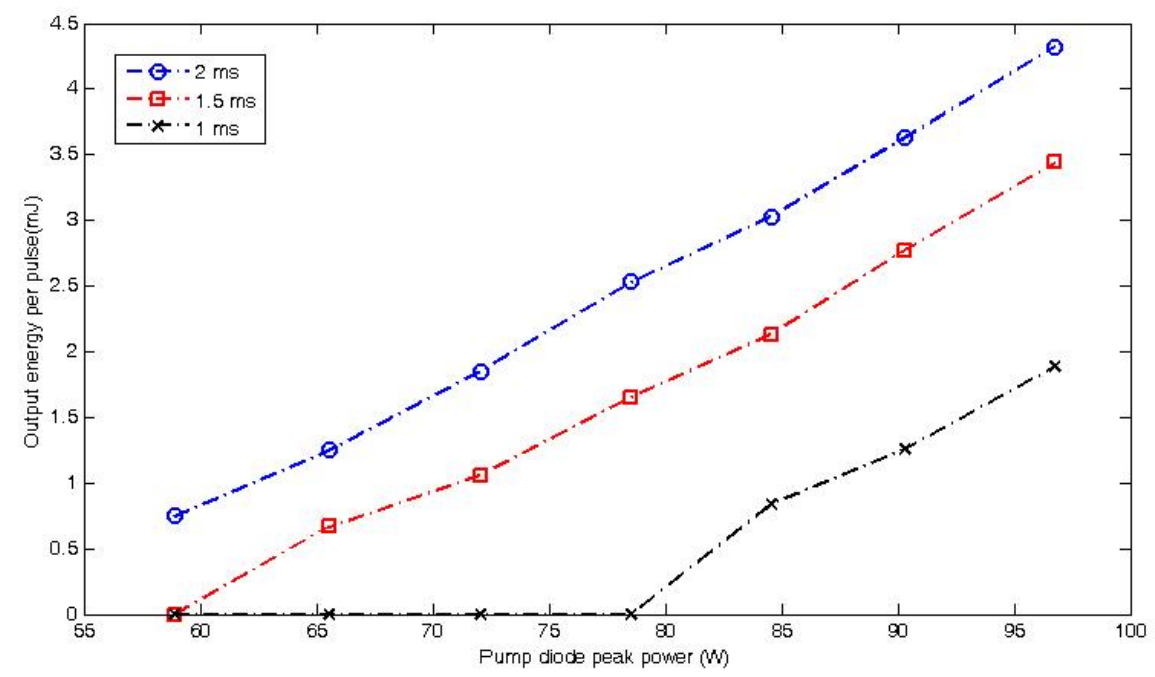

Figure 4.6: Regenerative amplifier output energy at $100 \mathrm{~Hz}$ as a function of peak pump power for the different pump pulse durations. 


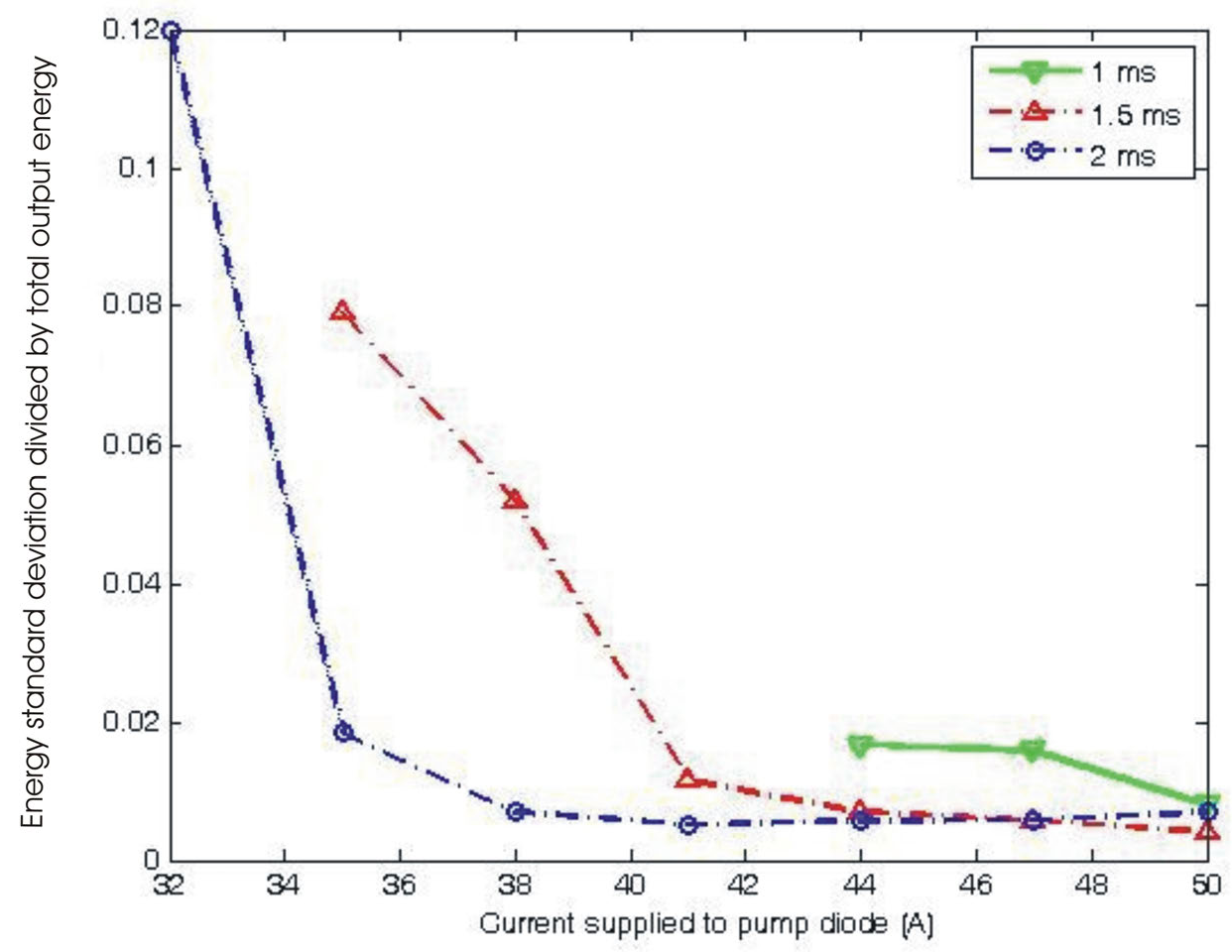

Figure 4.7: Relative pulse-to-pulse energy fluctuation of regenerative amplifier as a function of diode peak pump power and pump pulse duration.

no output energy variation data was recorded for the cavity when the crystal was not double passed, output energy was closer to one Joule and would vary by up to half of a Joule shot-to-shot. The drop down to tens of microJoules standard deviation is clearly an improvement, demonstrating another advantage of the double pass regenerative amplifier configuration.

Another parameter that was mentioned that will affect the usability of the laser is the transverse beam quality. An image of the beam has been made on a camera and is shown in Fig. 4.8. A fairly smooth and symetric transverse profile is evident. The output beam was monitered to be sure highly intense areas of the beam (hot spots) due to diffraction are not developing in the profile, especially as they could lead to optical damage in the following amplification stages. This beam profile has no evidence of hot spots. 


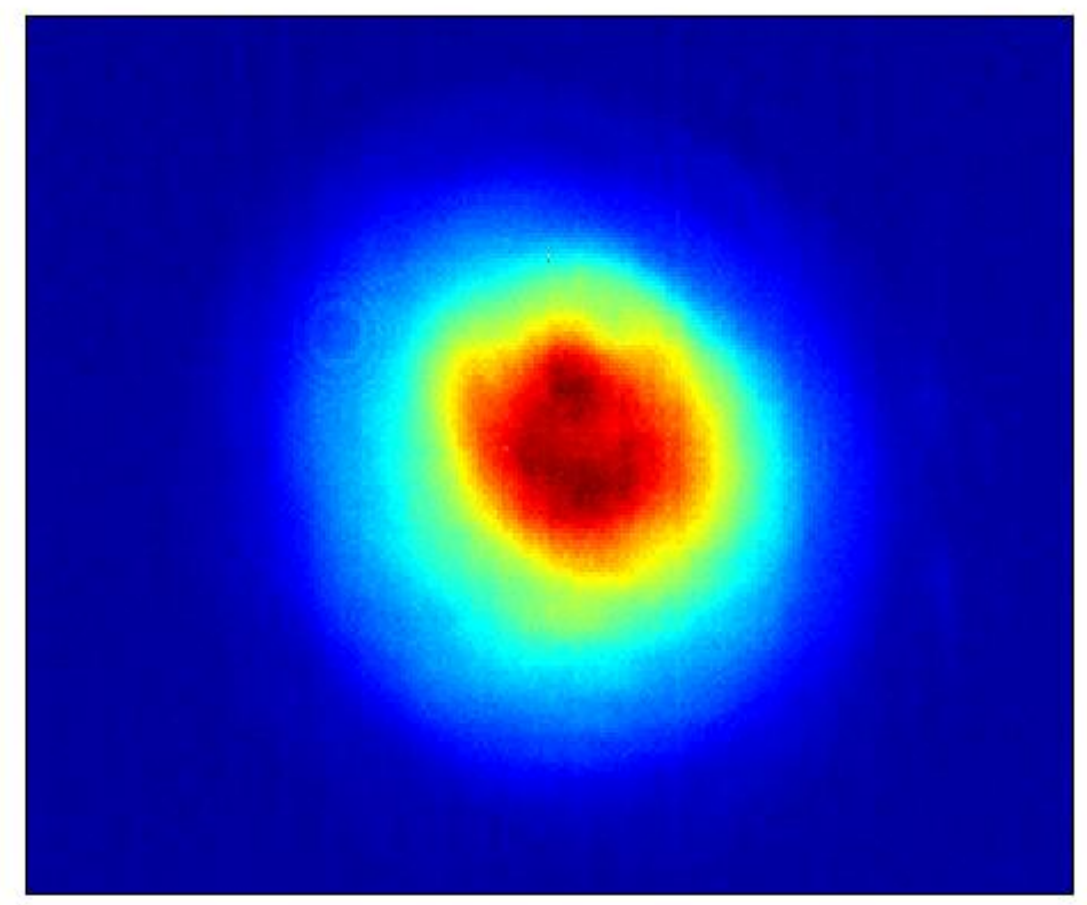

Figure 4.8: Image of the pulse transverse profile after the regenerative amplifier. 
The spectrum after amplification can be seen in Fig. 4.9, where an excellent spectral profile is evident. The spectrum can be shifted, modulated, and narrowed by a number of features in the amplifier. The closer the input spectrum from the stretcher is to the center of the room temperature Yb:YAG amplification spectrum, the wider the output spectrum will be, and the pulses will see more amplification resulting in higher pulse energy. This effect is complicated by the inter-cavity etalon which shifts the center wavelength of the regenerative amplifier cavity. The combination of these two factors make necessary an iterative process to maximize the output energy, spectral width, and most importantly to match the output center wavelength to the peak gain in the next cryo-amplification stage. The output pulse energy from the 2nd stage amplifier, which is discussed in the next chapter is more dependent on the input center wavelength than on the input pulse energy.

The pulse duration after the regenerative amplifier is once again recorded using second order autocorrelation. The results after compression can be seen in Fig. 4.10. The pulse duration after deconvolution is $3.6 \mathrm{ps}$ at the full width half maximum, when fit with a sech ${ }^{2}$ pulse shape. This is slightly longer than the transform limit for the bandwidth shown in figure 4.9 which is most likely do to a slight mismatch between the strecher and compressor.

\subsubsection{Future Improvements to 1st Stage Amplifier}

The regenerative amplifier output pulse energy can be increased by increasing the pump energy. Results of pumping with a 500 Watt diode can be seen in Fig. 4.11. Although the system is typically not operated this way, because the higher power diode is normally dedicated to pumping the second amplification stage, it can be seen that the regenerative amplifier is capable of increased amplification. A limiting factor here is optical damage from the higher intensity pulses as the pump energy is increased. In an earlier cavity designs, damage could be observed on a number of 


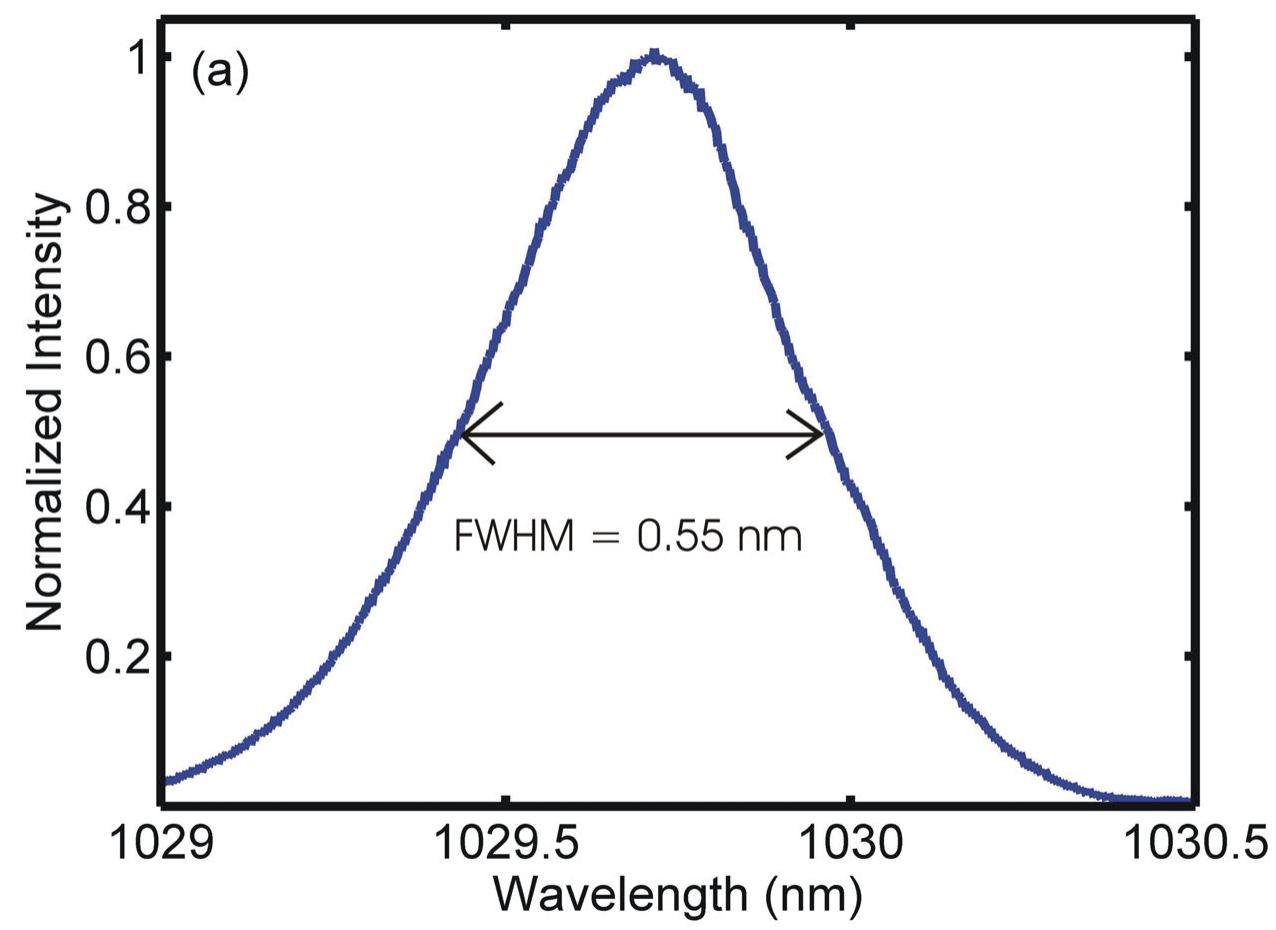

Figure 4.9: Bandwidth of the regenerative amplifier measured with a grating spectrometer. The full width at half maximum bandwidth is over half of a nanometer allowing for 2.8 ps transform limited pulses.

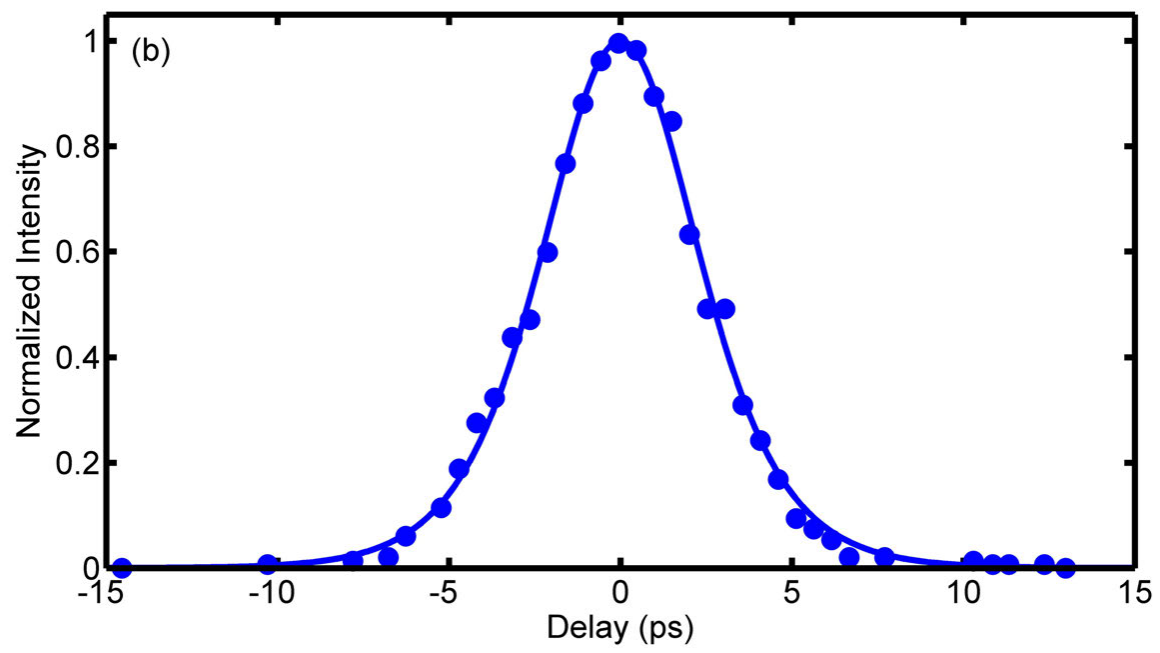

Figure 4.10: 2nd order autocorrelation of the regenerative amplifier compressed pulses. 


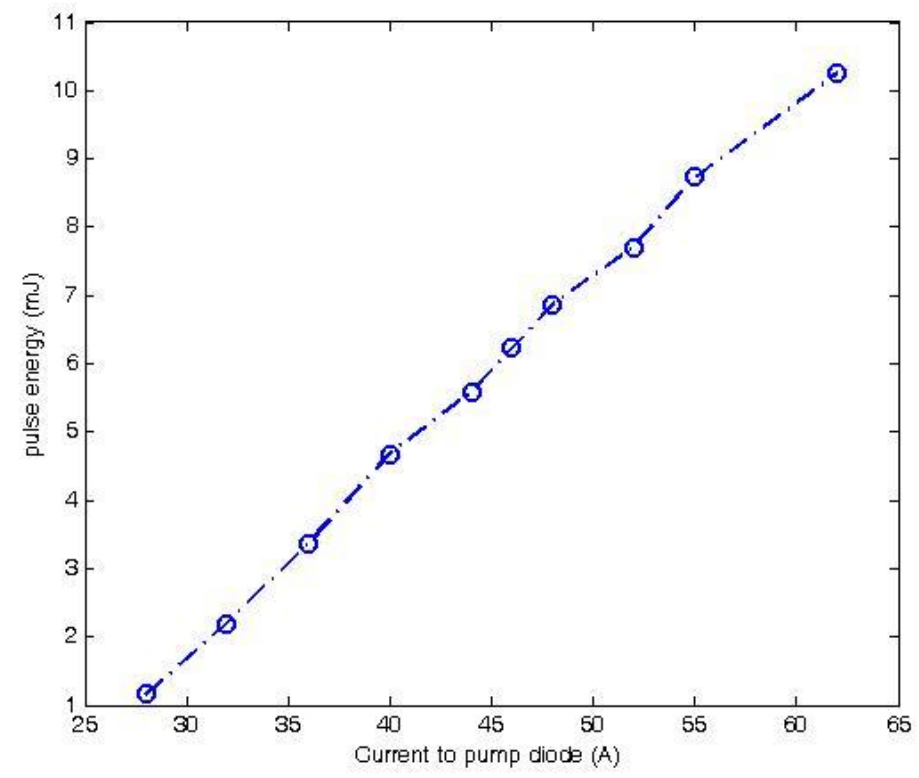

Figure 4.11: Regenerative amplifier pulse energy with $500 \mathrm{~W}$ diode as a function of pump diode current. Using a more powerful pump diode for the second stage amplifier, more energy can be extracted from the regenerative amplifier. Pulse energies in excess of $10 \mathrm{~mJ}$ with the current cavity brings the fluence dangerously close to damage threshold.

optical components as the output beam energy was increased to over $5 \mathrm{~mJ}$. For the current cavity layout and pump size, no optical damage was observed at $10 \mathrm{~mJ}$ where the fluence is close to $3.5 \mathrm{~J} / \mathrm{cm}^{2}$ on the crystal. This fluence is to some degree out of the comfort zone for long term operation. The fluence could be reduced by increasing the size of the mode on the crystal, but the decreased fluence would come at the cost of a reduced small signal gain. A Reduced small signal gain would result in more cavity passes in order to saturate the gain, which would result in more total cavity losses, and thus less efficient operation.

Another possible upgrade for the first stage amplifier would be to increase the repetition rate. Currently, increasing the repetition rate above $100 \mathrm{~Hz}$ results in a significant energy reduction. A possible solution would be to use a thinner crystal for more efficient cooling. A thinner crystal would require increased doping to store the same amount of energy for amplification. A thinner crystal can have possible 
deleterious effects such as transverse self amplified spontaneous emission losses also which was discussed above.

\subsubsection{Final Comments}

The regenerative amplifier is capable of reaching the desired $100 \mathrm{~Hz}$ repetition rate and achieves the energy goals of this stage of the system. It also produces a good optical quality beam, all of which is necessary to seed the next amplification stage. Additionally, because it is operated at room temperature, the bandwidth of the regenerative amplifier is sufficient to allow for the generation of 5 picosecond pulses after amplification in the folling high energy amplifier. 


\section{Chapter 5}

\section{Multi-pass amplifier}

\subsection{Amplifier Description and Optics}

The second stage amplifier is a multi-pass amplifier rather than a regenerative amplifier. Rather than coupling pulses into a cavity and passing through the gain region until reaching saturation, the pulses pass through the gain media a predetermined number of times based on alignment. The laser transverse diameter must be increased in this stage to reduce the peak fluence on the optics. Also the size of the gain media must be increased and doping is changed to account for the bigger beam.

A schematic of the second stage amplifier is shown in Fig. 5.1. Before entering the amplifier the beam is reshaped by a cylindrical imaging system to help account for astigmatism, and by a spherical imaging system to enlarge the beam size. The beam next proceeds through the isolation between the two amplifiers, and then passes four times through the cryogenically cooled two atomic percent Yb:YAG crystal. The size of the amplifier is slightly larger than if it were aligned to make two passes through the gain media and then sent back on itself, proceeding on a different path from the input by controlling the polarization in a similar fashion to what was previously mentioned. 


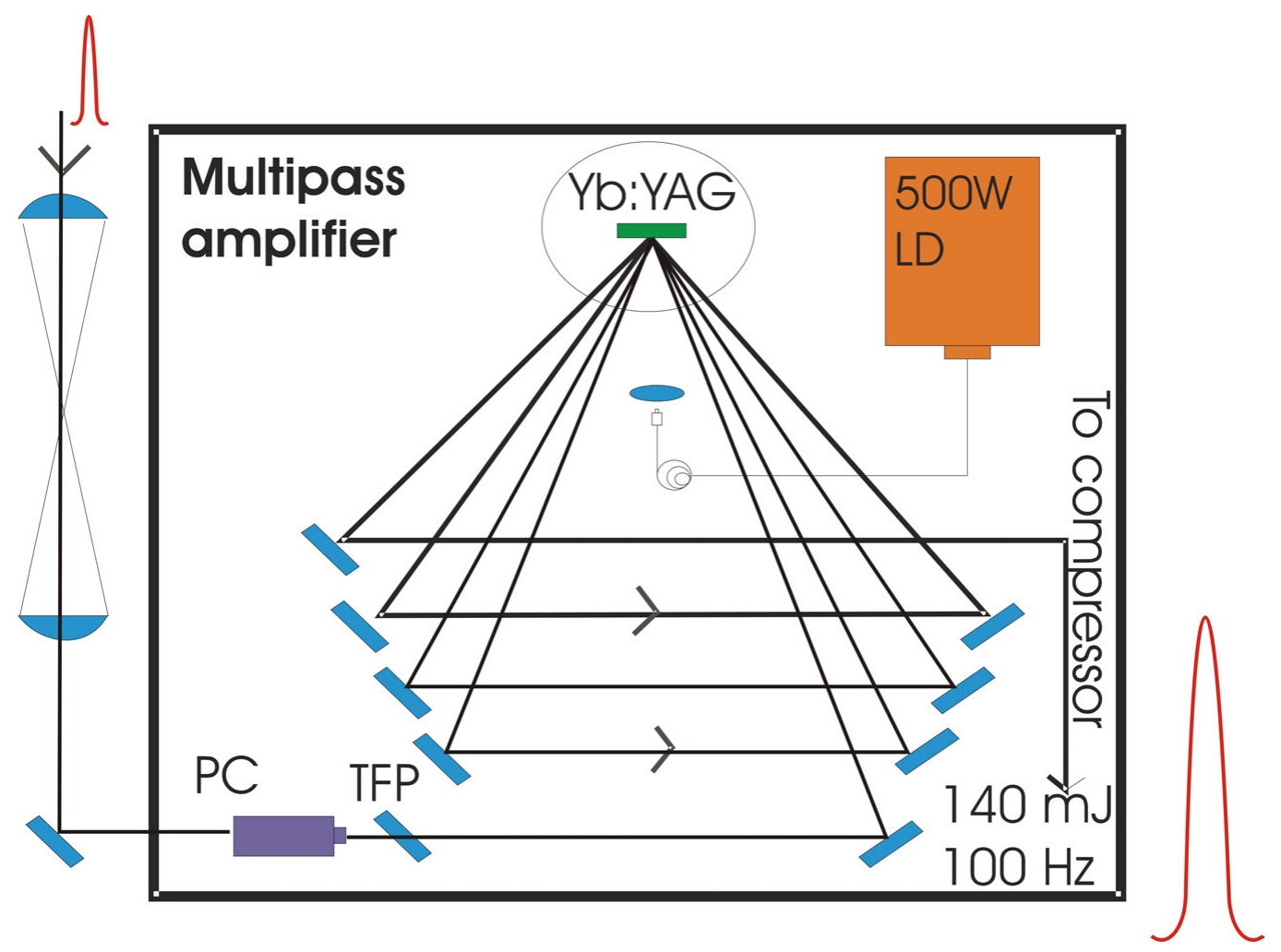

Figure 5.1: Schematic diagram of multipass cryogenically cooled Yb:YAG 2nd stage amplifier. PC, Pockels cell; TFP, thin film polarizer; LD, laser diode. 
There are at least two distinct advantages to using the adopted configuration. First, as can be seen from the figure, there are a number of 45 degree mirrors that are used in the amplifier. 45 degrees is around 10 degrees off Brewster's angle, which means that high reflectivity mirrors are more difficult to manufacture at this angle for light that has as P-polarization component. The non-send back alignment allows for the beam to remain S-polarized during all four passes through the amplifier.

A second advantage of not sending the beam back on itself is that no light can pass back towards the regenerative amplifier. If the polarization were shifted after two passes with a quarter waveplate before a send back mirror, and the beam were sent back on itself to be picked off by a polarizer after two additional passes, some light would inevitably leak through the polarizer and proceed towards the first stage amplifier because no polarizer is 100 percent efficient. These back propagation pulses would be further reduced in intensity by the isolation between the two amplification stages. It is important to note that even though very little energy propagates back, the regenerative amplifier is built to take picoJoule pulses and amplify them by 7 orders of magnitude, thus even small amounts of feedback from the second stage can still interrupt the amplification process. The geometrically separated four passes negate this effect.

The beam starts out with 2 millimeter diameter and by the time it leaves the amplifier, after $4 \mathrm{~m}$ of propegation, it has diverged to closer to 4 millimeters. Allowing the beam to diverge as it moves through the amplifier results in a preferable energy distribution for continued propagation through the system. The transverse profile of the pump image on the crystal is relatively flat top, therefore the energy in the center of the pump is extracted first to some degree, while the seed beam is smaller. As the beam diameter increases, more energy becomes available to amplify the outer part of the beam. the exiting beam approximates a flat top this way. This is preferable because while the gain can still be saturated, the center of the beam is less intense 

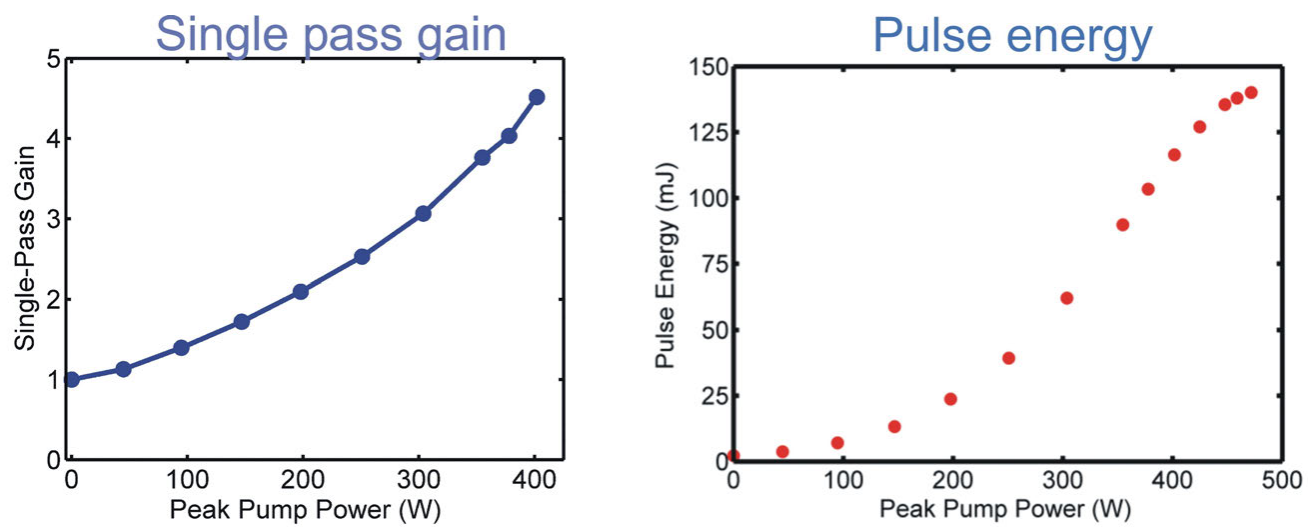

Figure 5.2: Right: Small signal gain for second stage amplifier. Left: Output pulse energy after all four passses.

than it would be if the seed and pump were mode-matched throughout amplification. This keeps the beam fluence below the damage threshold of the optical coatings. Additionally having a diverging beam reduces the chances that thermal lensing could damage mirrors or the crystal itself.

\subsection{Multi-pass Amplifier Performance}

A plot of the output pulse energy versus pump power can be seen in Fig. 5.2. This data was acquired using a 1.5 millisecond pump pulse with a peak power of 476 Watts impinging on the crystal. When pumped at the maximum peak pump power the amplifier reaches $140 \mathrm{~mJ}$. Fig. 5.2 shows that the amplifier opperates in a gainsaturation regime at full pump power. Additionally Fig. 5.2 shows the small signal gain for the amplifier after a single pass reaches 4.5 at $400 \mathrm{~W}$ of pump power.

An $M^{2}$ measurement of the beam is shown in Fig. 5.3. The $M^{2}$ of under 1.1 indicates excellent focusability. Here, 7 images of the beam were recorded at different distances from the focus. Based on the divergence of the beam the fit is finalized. No obvious hot spots or aberrations are seen in the beam profile also.

As expected, some small loss in the spectral bandwidth can be seen after amplification in the second stage. Figure 5.4 shows the output beam spectrum superimposed 


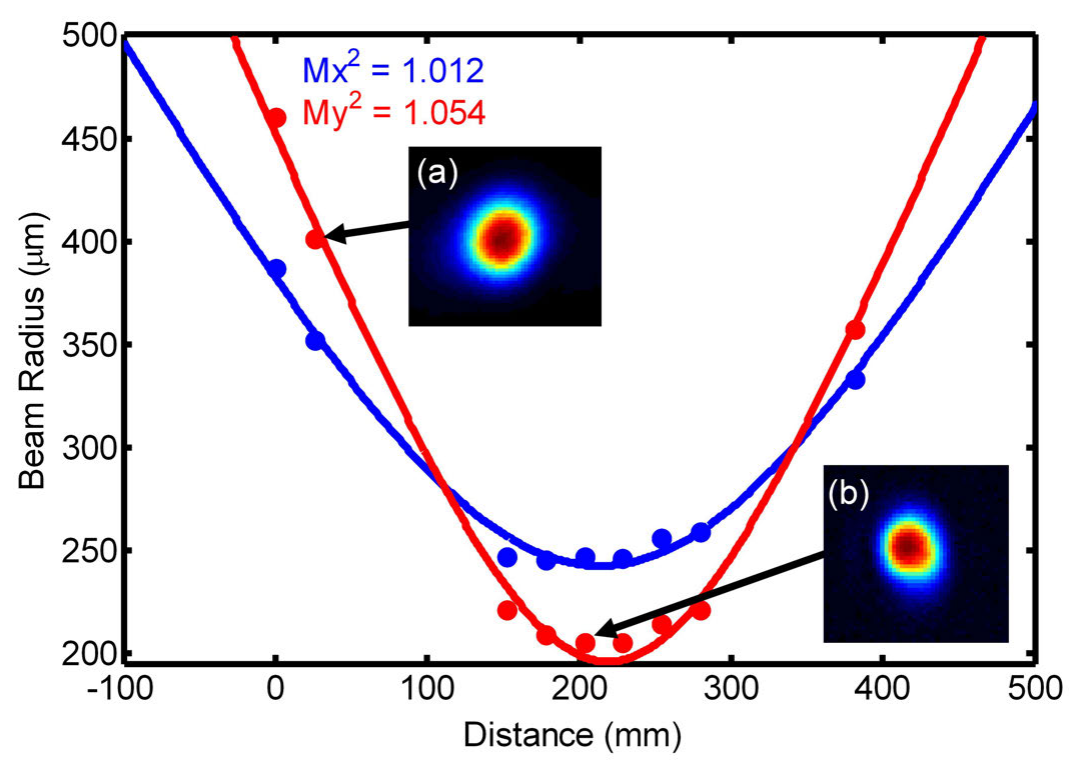

Figure 5.3: $M^{2}$ measurement for both axes of the beam after the 2nd stage amplifier. Some astigmatism is evident from the separation of the focal planes.

on the spectrum after the first amplifier. Clearly a few angstroms of bandwidth have been lost, which will result in a longer minimum pulse duration after compression. This behavior is not entirely unexpected because the crystal is cryo-cooled. Besides narrowing, the spectrum maintains a smooth unmodulated profile.

The second stage amplifier operates more efficiently than the other systems mentioned in section 1.5. The optical efficiency is close to $20 \%$ when the amplifier is pumped at full power producing $140 \mathrm{~mJ}$ pulses. This is a factor of 1.5 to 3 higher than other room temperature systems demonstrated with comparable energy. (??). The efficiency could be improved by multipassing the pump light through the crystal.

\subsection{Compressor}

The compressor makes use of a pair of dielectric diffraction grating pair ruled at 1780 lines per millimeter. The compressor is around 70 percent efficient. An imput of $140 \mathrm{~mJ}, 400 \mathrm{ps}$ pulses from the second stage produces $100 \mathrm{~mJ}, 4.8$ ps pulses at the 

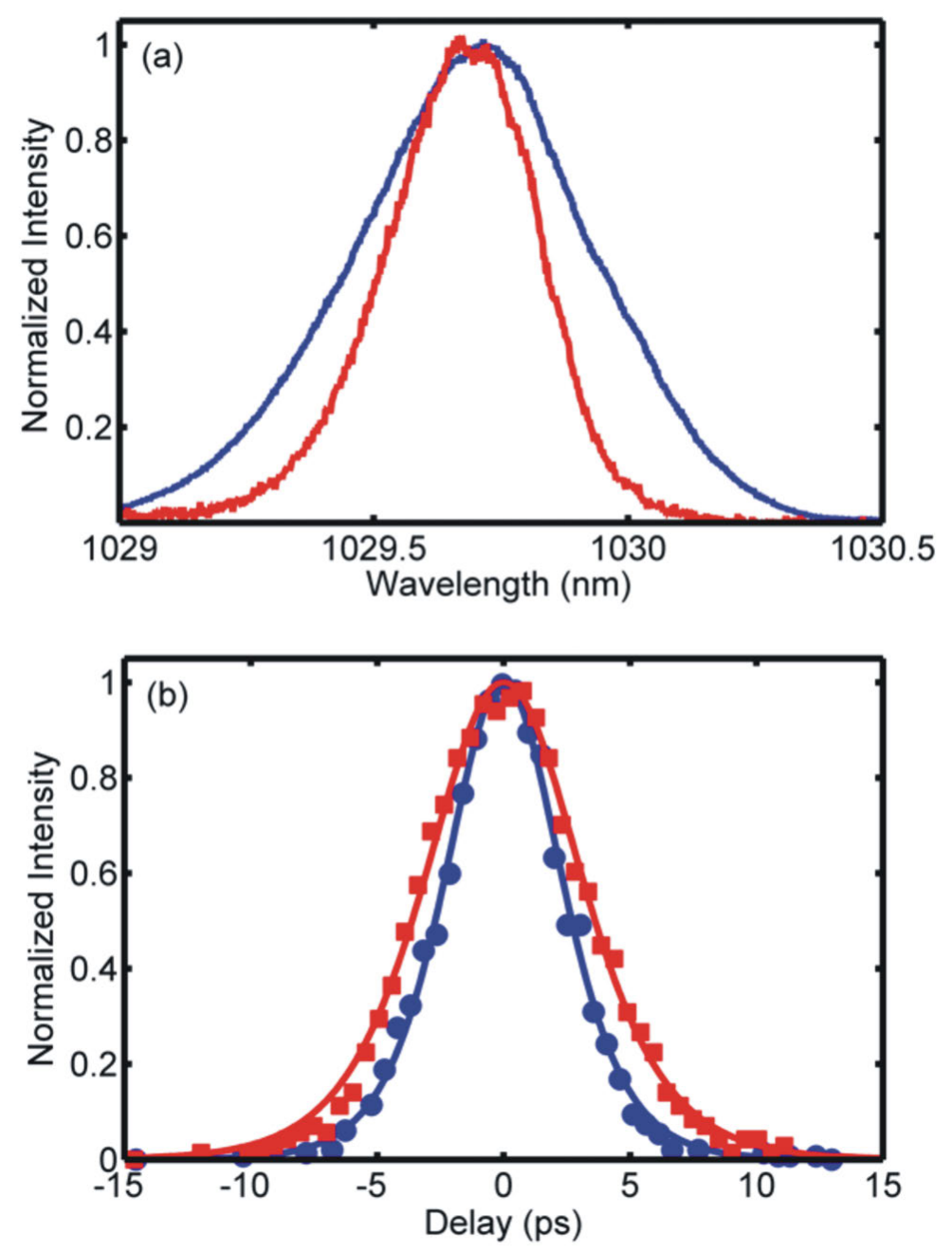

Figure 5.4: top: Gain narrowing from the cryo-cooled amplification stage is evident in the red spectral curve which is the spectrum after the 2 nd stage (blue is previously shown regen spectrum). bottom: Autocorrelation for both spectra, the 4.8 ps duration after the 2 nd stage is close to the 4.5 ps transform limit for a Gaussian. 
output. The compressor is kept in vacuum in order to avoid beam distorsion at high intensities. 


\section{Chapter 6}

\section{Conclusion}

In conclusion, I have demonstrated a hybrid room temperature/cryogenically cooled Yb:YAG chirped pulse amplification system. This all diode pumped system is able to produce $140 \mathrm{~mJ}$ pulses at $100 \mathrm{~Hz}$ repetition rate after two amplification stages. The pulse-to-pulse energy variations are characterized by a standard deviation of 0.3\%. After compression the $100 \mathrm{~mJ}$ pulses have a temporal duration of less than 5 ps. These pulses approach the transform-limit with a time-bandwidth product of 0.48. The selection of room temperature operation for the regenerative amplifier and cryo-cooled operation for the multi-pass amplification stage is aimed to maintain bandwidth during amplification in the stage with most gain, the regenerative amplifier, while decreasing saturation intensity and increasing hear dissipation for higher efficiency and average power in the second amplifier. At the maximum pump power the amplifier operates in the gain-saturation with an optical efficiency of $20 \%$. This optical efficiency exceeds those reported for room temperature diode-pumped amplifiers producing comparable energy by a factor of 1.5-3. Higher efficiency could be achieved by increasing the fraction of pump power absorbed by the Yb:YAG, increasing the doping, or double passing the pump light. Negligible thermal lensing is observed during the amplification process, and the beam shows good beam quality. 
The work reported in this thesis is motivated by the development of all-diodepumped soft x-ray lasers. Future work, in this area will require the addition of a third amplification stage to increase the energy to the Joule-level, as necessary to created the hot-dense plasmas necessary to obtain gain-saturated amplification in transitions of Ne-like and Ni-like. It is expected that the additional amplification by a factor of about 10, will not significantly narrow the bandwidth narrowing, allowing us to maintain pulse widths under $6 \mathrm{ps}$, that is near optimal to pump these type of lasers in for example the $18.9 \mathrm{~nm}$ line of Ni-like $\mathrm{Mo}$ and the $13.9 \mathrm{~nm}$ line of Ni-like Ag. Operation at repetition rates significantly higher than those that characterize the present $5-10 \mathrm{~Hz}$ table-top soft $\mathrm{x}$-ray lasers in that wavelength range would result in an increase in average power for applications requiring a large photon flux, such as nano-scale patterning or materials modification.

In its present configuration the system could also be used in other applications, such as damage threshold studies of multilayer coated optics, or to the pump for an optical parametric chirped pulse amplification laser system. 


\section{Bibliography}

[1] A. L. Schawlow and C. H. Townes, "Infrared and optical masers," Phys. Rev., vol. 112, pp. 1940-1949, Dec 1958.

[2] T. H. Maiman, "Stimulated optical radiation in ruby," Nature, vol. 187, pp. 493494.

[3] C. Das, A. Trivedi, K. Mitra, and T. Vo-Dinh, "Short pulse laser propagation through tissues for biomedical imaging," Journal of Physics D: Applied Physics, vol. 36, 2003.

[4] D. Parsons-Karavassilis, R. Jones, M. J. Cole, P. M. W. French, and J. R. Taylor, "Diode-pumped all-solid-state cr:lisgaf laser oscillator-amplifier system applied to laser ablation," Optics Communications, vol. 175, pp. 389-396.

[5] J. C. Chanteloup, D. Albach, A. Lucianetti, K. Ertel, S. Banerjee, D. Mason, Hernandez-Gomez, J. L. Collier, J. Hein, M. Wolf, J. Korner, and B. J. LeGarrec, "Multi kj level laser concepts for hiper facility," Journal of Physics Conference Series, vol. 244, 2010.

[6] D. H. Martz, D. Alessi, B. M. Luther, Y. Wang, D. Kemp, M. Berrill, and J. J. Rocca, "High-energy $13.9 \mathrm{~nm}$ table-top soft-x-ray laser at $2.5 \mathrm{hz}$ repetition rate excited by a slab-pumped ti:sapphire laser," Optics Letters, vol. 35, pp. 16321634. 
[7] A. Seigman, Lasers. University Science Books.

[8] S. Backus, C. G. Durfee, M. M. Murnane, and H. C. Kapteyn, "High power ultrafast lasers," Review of Scientific Instruments, vol. 69, pp. 1207-1223.

[9] J.-C. Diels and W. Rudolph, Ultrashort Laser Pulse Phenomena. Academic Press.

[10] http://en.wikipedia.org/wiki/Chirped_pulse_amplification.

[11] Z. Bor, "Distortion of femtosecond laser pulses in lenses," Opt. Lett., vol. 14, pp. 119-121, Jan 1989.

[12] E. Treacy, "Optical pulse compression with diffraction gratings," Quantum Electronics, IEEE Journal of, vol. 5, pp. 454 - 458, sep 1969.

[13] W. Koechner, Solid State Laser Engineering. Springer Sience.

[14] http://en.wikipedia.org/wiki/Flashlamp.

[15] W. F. Krupke, "Ytterbium solid-state lasers - the first decade," IEEE Journal On Selected Topics in Quantum Electronics, vol. 6, 2000.

[16] http://www.rp photonics.com/ytterbium_doped_gain_media.html.

[17] D. S. Sumida and T. Y. Fan, "effect of radiation trapping on fluorescence lifetime and emission cross section measurements in solid-state laser media," Optics Letters, vol. 19, p. 1343.

[18] R. L. Aggarwal, D. J. Ripin, J. R. Ochoa, and T. Y. Fan, "Measurement of thermo-optic properties of y3al5o12, lu3al5o12, yaio3, liyf4, liluf4, bay2f8, $\operatorname{kgd}($ wo4)2, and ky(wo4)2 laser crystals in the 80300k temperature range," Journal of Applied Physics, vol. 98, 2005. 
[19] L. D. DeLoach, S. A. Payne, L. L. Chase, L. K. Smith, W. L. Kway, and W. F. Krupke, "Evaluation of absorption and emission properties of $y b^{3+}$ doped crystals of laser applications," IEEE Journal of Quantum Electronics, vol. 29, 1994.

[20] A. A. Lagatsky, N. V. Kuleshov, and V. P. Mikhailov, "Diode-pumped cw lasing of yb:kyw and yb:kgw," Optics communications, vol. 165, pp. 71-75.

[21] J. Dong, M. Bass, Y. Mao, P. Deng, and F. Gan, "Dependence of the yb31 emission cross section and lifetime on temperature and concentration in yttrium aluminum garnet," J. Opt. Soc. Am. B, vol. 20, p. 1975.

[22] J. Tummler, R. Jung, H. Stiel, P. V. Nickles, and W. Sandner, "High-repetitionrate chirped-pulse-amplification thin-disk laser system with joule-level pulse energy," Optics Letters, vol. 34, pp. 1378-1380, 2009.

[23] T. Metzger, A. Schwarz, C. Y. Teisset, D. Sutter, A. Killi, R. Kienberger, and F. Krausz, "High-repetition-rate picosecond pump laser based on a yb:yag disk amplifier for optical parametric amplification," Optics Letters, vol. 34, pp. 2123$2125,2009$.

[24] S. Klingebiel, C. Wandt, C. Skrobol, I. Ahmad, S. A. Trushin, Z. Major, F. Krausz, and S. Karsch, "High energy picosecond yb:yag cpa system at 10 hz repetition rate for pumping optical parametric amplifiers," Optics Express, vol. 19, pp. 5357-5363, 2011.

[25] Y. Akahane, M. Aoyama, K. Ogawa, K. Tsuji, S. Tokita, J. Kawanaka, H. Nishioka, and K. Yamakawa, "High-energy, diode-pumped, picosecond yb:yag chirped-pulse regenerative amplifier for pumping optical parametric chirpedpulse amplification," Optics Letters, vol. 32, pp. 1899-1901, 2009.

[26] K. Hong, J. T. Gopinath, D. Rand, A. M. Siddiqui, S. Huang, E. Li, B. J. Eggleton, J. D. Hybl, T. Y. Fan, and F. X. Kartner, "High-energy, khz-repetition-rate, 
ps cryogenic yb:yag chirped-pulse amplifier," Optics Letters, vol. 35, pp. 17521754, 2010.

[27] F. J. Furch, B. A. Reagan, B. Luther, A. Curtis, S. P. Meehan, and J. Rocca, "Demonstration of an all-diode-pumped soft x-ray laser," Optics Letters, vol. 34, pp. $3352-3354,2009$.

[28] D. Attwood, Soft X-ray and Extreme Ultraviolet Radiation. Cambridge University Press.

[29] X. Yang, Z. zhan Xu, Y. xin Leng, H. he Lu, L. huang Lin, Z. quan Zhang, R. xin Li, W. qi Zhang, D. jun Yin, and B. Tang, "Multiterawatt laser system based on optical parametric chirped pulse amplification," Opt. Lett., vol. 27, pp. 1135-1137, 2002.

[30] I. N. Ross, J. L. Collier, P. Matousek, C. N. Danson, D. Neely, R. M. Allott, D. A. Pepler, C. Hernandez-Gomez, and K. Osvay, "Generation of terawatt pulses by use of optical parametric chirped pulse amplification," Appl. Opt., vol. 39, pp. 2422-2427, 2000.

[31] http://www.batop.de/information/SAM_infos.html\#Absorption].

[32] V. Pervak, C. Teisset, A. Sugita, S. Naumov, F. Krausz, and A. Apolonski, "High-dispersive mirrors for femtosecond lasers," Opt. Express, vol. 16, pp. 10220-10233, Jul 2008.

[33] R. Paschotta, J. Aus der Au, G. J. Spuhler, S. Erhard, A. Giesen, and U. Keller, "Passive mode locking of thin-disk lasers: effects of spatial hole burning," Applied Physics, pp. 267-278, 2001. 